\title{
NONSYMMETRIC COUPLING OF BEM AND MIXED FEM ON POLYHEDRAL INTERFACES
}

\author{
SALIM MEDDAHI, FRANCISCO-JAVIER SAYAS, AND VIRGINIA SELGÁS
}

\begin{abstract}
In this paper we propose and analyze some new methods for coupling mixed finite element and boundary element methods for the model problem of the Laplace equation in free space or in the exterior of a bounded domain. As opposed to the existing methods, which use the complete matrix of operators of the Calderón projector to obtain a symmetric coupled system, we propose methods with only one integral equation. The system can be considered as a further generalization of the Johnson-Nédélec coupling of BEM-FEM to the case of mixed formulations in the bounded domain. Using some recent analytical tools we are able to prove stability and convergence of Galerkin methods with very general conditions on the discrete spaces and no restriction relating the finite and boundary element spaces. This can be done for general Lipschitz interfaces and in particular, the coupling boundary can be taken to be a Lipschitz polyhedron. Both the indirect and the direct approaches for the boundary integral formulation are explored.
\end{abstract}

\section{INTRODUCTION}

Very early in the history of modern numerical analysis, the coupling of Finite and Boundary Element Methods was recognized to be a promising field for research and applications. The possibility of enjoying the best of both worlds [29] is an attractive feature of these coupled schemes. We can use the Boundary Element Method (BEM) to deal with unbounded regions where simple equations (linear, homogeneous, with constant coefficients) take place. We thus leave to the Finite Element Method (FEM) the task of dealing with source terms, nonlinearities, complex geometrical details, but relieve it of having to discretize huge regions where small variations happen. We also free the problem of modeling errors produced by cutting off an unbounded domain and devising approximate boundary conditions.

The first analytical approach to coupling of BEM and FEM appeared in 18, 4] and 5. It probably constitutes the first successful effort in the direction of a fully analyzed coupled numerical scheme, at least in the realm of variational methods for PDEs. The method of 18 ends up in a nonsymmetric system of operator equations, even though the original problem is the Laplace equation, which is clearly

Received by the editor May 13, 2009 and, in revised form, September 8, 2009.

2010 Mathematics Subject Classification. Primary 65N30.

Key words and phrases. Mixed FEM, BEM-FEM coupling, Lipschitz domains.

The first author was partially supported by the Spanish MEC Project MTM2007-65088.

The second author was partially supported by Spanish MEC Project MTM2007-63204 and Gobierno de Aragón (Grupo Consolidado PDIE).

The third author was partially supported the Spanish MEC project MTM2007-67596-C02-01 and Xunta de Galicia (PGIDIT07PXIB105257PR).

(c)2010 American Mathematical Society Reverts to public domain 28 years from publication 
symmetric. The proof in [18 uses the discrete Fredholm theorems that can be traced back to 20]. However, an important requirement for the applicability of this proof technique is the compactness of a certain integral operator that appears in the formulation. In the case of the Laplace equation, this operator is compact when the coupling boundary is smooth enough, but it is not for polygonal/polyhedral coupling interfaces. Obviously, this was considered as a drawback from the point of view of the finite element method, which is better applied on polygonal domains. In the case of the linear elasticity system, the smoothness of the interface is not enough to provide compactness of the corresponding integral operator and the method seemed not applicable in any situation. On smooth enough interfaces, a way around was found in 2 by using a nonstandard coupling in the pseudostress.

The smoothness requirement was not a deterrent for the development of this kind of coupled BEM-FEM formulations (called one-equation couplings in [13), with extensions to the Stokes system [28] and to nonlinear problems [13], or with the use of curved triangles [24]. However, smoothness of the interface was generally considered to be a serious flaw in the method.

A breakthrough occurred in the late 1980s with the appearance of the symmetric coupling techniques in [9] and [19. This was the starting point of an expansion of coupling techniques of BEM with other FEM schemes: Raviart-Thomas elements in 25, more general mixed FEM in 6], Crouzeix-Raviart elements in 7, discontinuous Galerkin methods [16, 15, etc. A general frame where most of these methods fit is given in [8]. Symmetric coupling (or, in the terminology of [13], two-equation coupling) has been considered the standard solution to avoid smooth interfaces; although, it must be said, no practical problems of the original Johnson-Nédélec type schemes have been reported to the best of the authors' knowledge.

The proof in 27] of the validity of the Johnson-Nédélec approach for any Lipschitz coupling interface marks a new inflection point in this direction. The paper 27. follows an idea originally designed in 22] to deal with simultaneous discrete analysis of symmetric BEM-FEM schemes for the operator equation $-\Delta u+s^{2} u=f$ for all $s \in \mathbb{C}$ with $\operatorname{Re} s>0$. In 22 , the need to work in the entire resolvent set for the Laplace operator precluded the use of discrete Fredholm theorems and they were obliged to rethink what it means to discretize a boundary integral equation, understanding this process as an equivalent problem in free space with somewhat exotic transmission conditions across the boundary. The results in 27] have been expanded in [14] to extend the stability results of the quasi-symmetric coupling of Bielak and MacCamy [2 to any Lipschitz interface.

In this paper we propose to create and analyze a simple nonsymmetric oneequation coupling of mixed FEM with BEM. We will restrict our attention to the three-dimensional Laplace equation but we will work in great generality with the discrete spaces proving essentially that any choice works, as long as the mixed FEM pair of spaces provide a stable method.

Some words on how symmetric and nonsymmetric couplings of BEM and FEM compare seem pertinent at this point. The analysis of symmetric coupling schemes for BEM and FEM is always based upon an ellipticity principle. For the coupled equations to become symmetric, it is compulsory to change the sign of one or more of the rows of the system of operator equations. This change makes the system strongly indefinite. Symmetric methods benefit from the wider availability of linear solvers for symmetric systems. This advantage is lost when what is symmetric 
is the principal part of the operator, as when we apply these ideas to the Helmholtz equation. Another drawback of symmetric couplings is the need to use (and discretize) all integral operators of the Calderón projector. Furthermore, symmetric couplings follow from the clever artifice of using all the Calderón identities whereas nonsymmetric couplings are natural applications of one of the variants of Green's Third Identity. This additional simplicity of the formulation makes nonsymmetric couplings preferred in many practical situations. Finally, from the point of view of storage, if the full matrices are never assembled (but only its relevant blocks are built and stored), the number of submatrices to keep in nonsymmetric formulations is equal to or smaller than in symmetric formulations. This is due to the fact that less integral operators are employed in the nonsymmetric case.

The paper is structured as follows. In Section 2 we explain two formulations (with direct and indirect boundary integral equations) for the transmission problem in free space, and we give hypotheses for their Galerkin discretization. In this section we state the main well posedness and stability results of this paper. In Sections 3 and 4 we give the proof of these theorems. In particular, the discrete results will be proved in more generality than initially needed, with no additional effort. This increased generality is made the most of when we notice that imposition of Neumann boundary conditions on an interior boundary (Section 5) is just a particular case of what has been done in free space. The case of Dirichlet conditions deserves special attention (Section 6) and the result is slightly different, although its quality (asymptotic stability for general methods with any interface) is essentially the same. In Section 7 we report some numerical experiments with a particular choice of spaces.

Throughout this paper we will be loosely using elementary results on the Sobolev spaces $H^{1}(\Omega), H^{ \pm 1 / 2}(\Gamma)$ and $\mathbf{H}(\operatorname{div}, \Omega)$, results that can be considered as part of the folklore shared by numerical analysts of partial differential equations. A common reference for Sobolev spaces theory is [1]. For $\mathbf{H}(\operatorname{div}, \Omega)$ type spaces, 17] is the usual choice. Some very basic weighted Sobolev spaces (identifiable with BeppoLevi spaces) will also be used. An easy to find reference for the most elementary properties of these is 26 . The theory of boundary integral operators for the Laplace equation on a Lipschitz domain appears in 10, and can be found extended in great generality in [23], which is also an excellent reference for Sobolev spaces on domains with Lipschitz boundaries and on these boundaries.

\section{MAin RESUlts}

The aim of this paper is the solution of the Laplace equation in free space with compactly supported source terms and discontinuous transmission data across an interface that surrounds the sources. Modifications for the case when we impose boundary conditions on an interior boundary will be dealt with in Sections 5 and 6. In this section we will only state results. Proofs are postponed to Sections 3 and 4.

We consider a bounded domain $\Omega \subset \mathbb{R}^{3}$, with Lipschitz boundary $\Gamma$ and connected exterior $\Omega^{e}:=\mathbb{R}^{3} \backslash \bar{\Omega}$. The normal vector on $\Gamma$ is pointed outwards. Traces and normal derivatives on $\Gamma$ of functions defined on $\Omega$ will be denoted with the symbols $\gamma$ and $\partial_{\boldsymbol{\nu}}$, respectively. When the function is defined in the exterior domain $\Omega^{e}$, the superscript $e$ will be added to the symbols $\gamma^{e}$ and $\partial_{\nu}^{e}$. We will use 
the jump operators for functions defined in $\Omega \cup \Omega^{e}$ :

$$
[\gamma u]:=\gamma u-\gamma^{e} u, \quad\left[\partial_{\nu} u\right]:=\partial_{\nu} u-\partial_{\nu}^{e} u .
$$

Boldface symbols will be used for vector fields. The normal component of $\mathbf{p}: \Omega \rightarrow$ $\mathbb{R}^{3}$ on $\Gamma$ is denoted

$$
\gamma_{\nu} \mathbf{p}:=\mathbf{p} \cdot \boldsymbol{\nu}
$$

The transmission problem we will be dealing with is

$$
\begin{array}{ll}
\mathbf{p}-\nabla u=0 \quad \text { in } \Omega, & -\operatorname{div} \mathbf{p}=f \quad \text { in } \Omega, \\
{[\gamma u]=\beta_{0},} & \gamma_{\boldsymbol{\nu}} \mathbf{p}=\partial_{\boldsymbol{\nu}}^{e} u+\beta_{1}, \\
-\Delta u=0 \quad \text { in } \Omega^{e}, & \\
u=\mathcal{O}\left(|\mathbf{x}|^{-1}\right) \text { as }|\mathbf{x}| \rightarrow \infty . &
\end{array}
$$

Apart from the usual $H^{1}(\Omega)$ Sobolev spaces, we will use the weighted space

$$
W^{1}\left(\Omega^{e}\right):=\left\{u: \Omega^{e} \rightarrow \mathbb{R}: \rho u \in L^{2}\left(\Omega^{e}\right), \quad \nabla u \in\left(L^{2}\left(\Omega^{e}\right)\right)^{3}\right\},
$$

where $\rho(\mathbf{x}):=1 / \sqrt{1+|\mathbf{x}|^{2}}$. For basic properties of this space, see [26, Section 2.5]. If

$$
f \in L^{2}(\Omega), \quad \beta_{0} \in H^{1 / 2}(\Gamma), \quad \beta_{1} \in H^{-1 / 2}(\Gamma),
$$

then (11) admits a unique solution $u \in H^{1}(\Omega) \times W^{1}\left(\Omega^{e}\right) \cong W^{1}\left(\Omega \cup \Omega^{e}\right)$. (We will adopt the convention of identifying a function defined on $\Omega \cup \Omega^{e}$ with a pair of functions defined on the respective regions.) A proof of this existence and uniqueness result derives from the treatment of this problem in its primal form ( $\mathbf{p}$ is absent from the formulation) in [27, Section 4].

Let $\Phi(r):=1 /(4 \pi r)$ be the fundamental solution of the three-dimensional Laplace operator. With it we construct the single and double layer potentials:

$$
\begin{aligned}
\mathrm{S} \lambda & :=\int_{\Gamma} \Phi(|\cdot-\mathbf{y}|) \lambda(\mathbf{y}) \mathrm{d} \Gamma(\mathbf{y}), \\
\mathrm{D} \varphi & :=\int_{\Gamma} \partial_{\boldsymbol{\nu}(\mathbf{y})} \Phi(|\cdot-\mathbf{y}|) \varphi(\mathbf{y}) \mathrm{d} \Gamma(\mathbf{y}) .
\end{aligned}
$$

The integral expressions of these potentials can be extended in the following way: given $\lambda \in H^{-1 / 2}(\Gamma)$ and $\varphi \in H^{1 / 2}(\Gamma), \mathrm{S} \lambda$ and $\mathrm{D} \varphi$ define functions in $H^{1}(\Omega) \times$ $W^{1}\left(\Omega^{e}\right)$ that solve Laplace's equation in $\Omega \cup \Omega^{e}$. A rigorous introduction of these layer potentials with Lipschitz regularity of the boundaries was carried out in [10] and can be found extended in great generality to other elliptic systems in [23]. For any forthcoming result on layer potentials and boundary integral operators, the reader is referred to [10] and 23]. Note that neither of these references use the weighted Sobolev space, remaining with local $H^{1}$ behavior instead. However, since potentials define smooth functions away from the boundary $\Gamma$, and the weight affects only integrability properties at infinity, the correct integral behavior is easily proved. Green's Third Identity can be stated as follows: if $u \in H^{1}(\Omega) \times W^{1}\left(\Omega^{e}\right)$ satisfies $\Delta u=0$ in $\Omega \cup \Omega^{e}$, then

$$
u=\mathrm{S}\left[\partial_{\nu} u\right]-\mathrm{D}[\gamma u] .
$$

Note that, in particular,

$$
\mathrm{D} 1= \begin{cases}-1, & \text { in } \Omega, \\ 0, & \text { in } \Omega^{e} .\end{cases}
$$


TABLE 1. The jump relations of potentials: the upper row are the operators of restriction to the boundary, which applied to the potentials $\mathrm{S}$ and $\mathrm{D}$ give the boundary operators in the table.

\begin{tabular}{|c||c|c|c|c|c|c|}
\hline & $\gamma$ & $\gamma^{e}$ & $\partial_{\boldsymbol{\nu}}$ & $\partial_{\boldsymbol{\nu}}^{e}$ & {$[\gamma]$} & {$\left[\partial_{\boldsymbol{\nu}}\right]$} \\
\hline $\mathrm{S}$ & $\mathrm{V}$ & $\mathrm{V}$ & $\frac{1}{2} \mathrm{I}+\mathrm{K}^{t}$ & $-\frac{1}{2} \mathrm{I}+\mathrm{K}^{t}$ & 0 & $\mathrm{I}$ \\
\hline $\mathrm{D}$ & $-\frac{1}{2} \mathrm{I}+\mathrm{K}$ & $\frac{1}{2} \mathrm{I}+\mathrm{K}$ & $-\mathrm{W}$ & $-\mathrm{W}$ & $-\mathrm{I}$ & 0 \\
\hline
\end{tabular}

We will make repeated use of the following integral operators, defining functions on $\Gamma$ for a given density $\varphi$ :

$$
\begin{aligned}
\mathrm{K} \varphi & :=\int_{\Gamma} \partial_{\boldsymbol{\nu}(\mathbf{y})} \Phi(|\cdot-\mathbf{y}|) \varphi(\mathbf{y}) \mathrm{d} \Gamma(\mathbf{y}), \\
\mathrm{W} \varphi & :=-\partial_{\boldsymbol{\nu}} \int_{\Gamma} \partial_{\boldsymbol{\nu}(\mathbf{y})} \Phi(|\cdot-\mathbf{y}|) \varphi(\mathbf{y}) \mathrm{d} \Gamma(\mathbf{y}) .
\end{aligned}
$$

The operator $\mathrm{K}: H^{1 / 2}(\Gamma) \rightarrow H^{1 / 2}(\Gamma)$ is bounded and we will denote its transpose $\mathrm{K}^{t}: H^{-1 / 2}(\Gamma) \rightarrow H^{-1 / 2}(\Gamma)$. The so-called hypersingular operator $\mathrm{W}: H^{1 / 2}(\Gamma) \rightarrow$ $H^{-1 / 2}(\Gamma)$ is also bounded. With these operators, the limiting values of the layer potentials and their jumps across $\Gamma$ can be described. This is done in Table 1 for ease of reference. There is an additional operator in this table, namely $\mathrm{V}$, which is defined with the same integral expression as the potential $\mathrm{S}$, restricted to points on the boundary.

2.1. Coupling with a direct boundary integral method. The first proposal for a coupled formulation uses Green's Third Identity in the exterior domain. We will take

$$
\psi:=\gamma^{e} u \in H^{1 / 2}(\Gamma)
$$

as unknown. We can then write for the solution of (1),

$$
u=\mathrm{D} \gamma^{e} u-\mathrm{S} \partial_{\nu}^{e} u=\mathrm{D} \psi-\mathrm{S}\left(\gamma_{\boldsymbol{\nu}} \mathbf{p}-\beta_{1}\right), \quad \text { in } \Omega^{e} .
$$

This is just (2) taking $u \equiv 0$ in the interior domain. Using the jump relations (Table 11) we have the following identity:

$$
\left(\frac{1}{2} \mathrm{I}+\mathrm{K}\right)^{t} \gamma_{\boldsymbol{\nu}} \mathbf{p}+\mathrm{W} \psi=\left(\frac{1}{2} \mathrm{I}+\mathrm{K}\right)^{t} \beta_{1} .
$$

A coupled formulation of (1) is obtained when we propose a standard mixed formulation of the interior problem and use the fact that $\gamma u=\psi+\beta_{0}$. The natural space for $\mathbf{p}$ is

$$
\mathbf{H}(\operatorname{div}, \Omega):=\left\{\mathbf{p} \in\left(L^{2}(\Omega)\right)^{3}: \operatorname{div} \mathbf{p} \in L^{2}(\Omega)\right\} .
$$

For properties of this space we refer to [17. The weak formulation leads to the following problem: find $(\mathbf{p}, u, \psi) \in \mathbf{H}(\operatorname{div}, \Omega) \times L^{2}(\Omega) \times H^{1 / 2}(\Gamma)$ such that

$$
\begin{array}{ll}
(\mathbf{p}, \mathbf{q})_{\Omega}+(u, \operatorname{div} \mathbf{q})_{\Omega}-\left\langle\gamma_{\nu} \mathbf{q}, \psi\right\rangle=\left\langle\gamma_{\nu} \mathbf{q}, \beta_{0}\right\rangle, & \forall \mathbf{q} \in \mathbf{H}(\operatorname{div}, \Omega), \\
(\operatorname{div} \mathbf{p}, v)_{\Omega}=-(f, v)_{\Omega}, & \forall v \in L^{2}(\Omega), \\
\left\langle\left(\frac{1}{2} \mathrm{I}+\mathrm{K}\right)^{t} \gamma_{\nu} \mathbf{p}, \varphi\right\rangle+\langle\mathrm{W} \psi, \varphi\rangle=\left\langle\left(\frac{1}{2} \mathrm{I}+\mathrm{K}\right)^{t} \beta_{1}, \varphi\right\rangle, & \forall \varphi \in H^{1 / 2}(\Gamma) .
\end{array}
$$


In (7) we have used the notation

$$
(\mathbf{p}, \mathbf{q})_{\Omega}:=\int_{\Omega} \mathbf{p} \cdot \mathbf{q}, \quad(u, v)_{\Omega}:=\int_{\Omega} u v
$$

and the angled brackets to denote the duality product of $H^{-1 / 2}(\Gamma)$ with $H^{1 / 2}(\Gamma)$. As the following result states, this formulation leads to a solvable problem, with a one-dimensional kernel. We will show later how to modify this problem to obtain a well-posed system and how to choose the right solution from the system (note that (11) is uniquely solvable even when (7) is not).

Theorem 1. Equations (7) are solvable in $\mathbf{H}(\operatorname{div}, \Omega) \times L^{2}(\Omega) \times H^{1 / 2}(\Gamma)$ for the given right-hand side. Its solution is $\left(\mathbf{p}, u+c, \gamma^{e} u+c\right)$ where $(u, \mathbf{p})$ is the solution of (11) and $c$ is an arbitrary constant.

2.2. Coupling with an indirect boundary integral method. An alternative method consists of using a double layer potential to represent the exterior component of $u$. However, this potential 'ansatz' imposes a restriction on the data, because of the following fact: if $u \in W^{1}\left(\Omega^{e}\right)$ satisfies $\Delta u=0$ in $\Omega^{e}$, then

$$
u=\mathrm{D} \xi \text { with } \xi \in H^{1 / 2}(\Gamma) \quad \Longleftrightarrow \quad\left\langle\partial_{\nu}^{e} u, 1\right\rangle=0 .
$$

Therefore, if we are going to use this type of formulation, we need

$$
\left\langle\beta_{1}, 1\right\rangle=\left\langle\gamma_{\boldsymbol{\nu}} \mathbf{p}, 1\right\rangle=(\operatorname{div} \mathbf{p}, 1)_{\Omega}=-(f, 1)_{\Omega},
$$

that is,

$$
(f, 1)_{\Omega}+\left\langle\beta_{1}, 1\right\rangle=0 .
$$

Note that D1 $\equiv 0$ in $\Omega^{e}$ (see (3) ) and therefore we cannot expect a uniquely solvable system with $\xi$ as an unknown.

Using the jump relations (Table 1) we can translate the transmission conditions to these two simple equations:

$$
\gamma u=\left(\frac{1}{2} \mathrm{I}+\mathrm{K}\right) \xi+\beta_{0}, \quad \gamma_{\boldsymbol{\nu}} \mathbf{p}+\mathrm{W} \xi=\beta_{1} .
$$

The second of these conditions will remain as one of the equations of the coupled system, whereas the first one will be incorporated as a natural condition in the mixed formulation for the interior domain. In a first approach, we arrive at the variational problem: find $(\mathbf{p}, u, \xi) \in \mathbf{H}(\operatorname{div}, \Omega) \times L^{2}(\Omega) \times H^{1 / 2}(\Gamma)$ such that

$$
\begin{array}{ll}
(\mathbf{p}, \mathbf{q})_{\Omega}+(u, \operatorname{div} \mathbf{q})_{\Omega}-\left\langle\gamma_{\nu} \mathbf{q},\left(\frac{1}{2} \mathrm{I}+\mathrm{K}\right) \xi\right\rangle=\left\langle\gamma_{\boldsymbol{\nu}} \mathbf{q}, \beta_{0}\right\rangle, & \forall \mathbf{q} \in \mathbf{H}(\operatorname{div}, \Omega), \\
(\operatorname{div} \mathbf{p}, v)_{\Omega}=-(f, v)_{\Omega}, & \forall v \in L^{2}(\Omega), \\
\left\langle\gamma_{\nu} \mathbf{p}, \varphi\right\rangle+\langle\mathrm{W} \xi, \varphi\rangle=\left\langle\beta_{1}, \varphi\right\rangle, & \forall \varphi \in H^{1 / 2}(\Gamma) .
\end{array}
$$

Theorem 2. Equations (9) are solvable in $\mathbf{H}(\operatorname{div}, \Omega) \times L^{2}(\Omega) \times H^{1 / 2}(\Gamma)$ for the given right-hand side if and only if (18) holds. Its solution is $(\mathbf{p}, u, \xi+c)$ where $(u, \mathbf{p})$ is the solution of (1), $c$ is an arbitrary constant and $u=\mathrm{D}(\xi+c)$ is the exterior component of the solution.

Let us briefly compare problems (7) and (9). Up to a change of sign of the third row and column, both systems are mutually transposed. This is often the case in problems that involve boundary integral equations: one operator equation corresponds to the direct approach and the transpose to the indirect approach. The right-hand side of the indirect approach (9) is simpler and data do not appear under the action of integral operators. Moreover, the integral representation of the 
exterior problem is just a potential, much simpler than the representation (16) for the exterior problem. On the other hand, the direct approach always gives a solvable system (there is no compatibility condition (8) on the data) and the unknown on the boundary is part of the solution, whereas $\xi$ in (9) is just a density with no further meaning. The lack of uniqueness is going to be simpler to deal with in the indirect case, which shows another advantage of this formulation. However, it has to be recognized that the engineering community favors direct formulations, which gives these a leading role in practical applications.

The lack of uniqueness in the density can be easily mended by looking for the third component in

$$
H_{0}^{1 / 2}(\Gamma):=\left\{\xi \in H^{1 / 2}(\Gamma):\langle 1, \xi\rangle=0\right\} .
$$

The reduced formulation consists of looking for the solution of (9) in $\mathbf{H}(\operatorname{div}, \Omega) \times$ $L^{2}(\Omega) \times H_{0}^{1 / 2}(\Gamma)$ and testing these equations only in the same space. This is stated in the following result.

Theorem 3. Let $\ell_{1} \in \mathbf{H}(\operatorname{div}, \Omega)^{\prime}, \ell_{2} \in L^{2}(\Omega)^{\prime}$ and $\ell_{3} \in H_{0}^{1 / 2}(\Gamma)^{\prime}$. The following problem is well posed: find $(\mathbf{p}, u, \xi) \in \mathbf{H}(\operatorname{div}, \Omega) \times L^{2}(\Omega) \times H_{0}^{1 / 2}(\Gamma)$ such that

$$
\begin{array}{ll}
(\mathbf{p}, \mathbf{q})_{\Omega}+(u, \operatorname{div} \mathbf{q})_{\Omega}-\left\langle\gamma_{\nu} \mathbf{q},\left(\frac{1}{2} \mathrm{I}+\mathrm{K}\right) \xi\right\rangle=\ell_{1}(\mathbf{q}), & \forall \mathbf{q} \in \mathbf{H}(\operatorname{div}, \Omega), \\
(\operatorname{div} \mathbf{p}, v)_{\Omega}=\ell_{2}(v), & \forall v \in L^{2}(\Omega), \\
\left\langle\gamma_{\nu} \mathbf{p}, \varphi\right\rangle+\langle\mathrm{W} \xi, \varphi\rangle=\ell_{3}(\varphi), & \forall \varphi \in H_{0}^{1 / 2}(\Gamma) .
\end{array}
$$

If the right-hand side is the same as (9), we obtain one of the solutions of (9) and, through it, the solution of the transmission problem (1).

When condition (8) is not satisfied, we can rewrite the problem in the unknowns

$$
v:=u-\frac{(f, 1)_{\Omega}+\left\langle\beta_{1}, 1\right\rangle}{\langle 1,1\rangle} \mathrm{S} 1, \quad \mathbf{q}:=\nabla v .
$$

This change of unknowns affects only $\beta_{1}$ ( $f$ and $\beta_{0}$ are not modified) and the new data comply with (8).

2.3. A reduced coupling with the direct method. We can try to select one of the solutions of (77) using the same technique as in Theorem 3. The main difference is going to be the fact that this simple choice yields one of the solutions of (7) that might not be the solution to (11) and we need some small postprocessing step to modify a constant value in the interior. The average operator on the boundary

$$
\mathrm{J} \varphi:=\frac{\langle 1, \varphi\rangle}{\langle 1,1\rangle}=\frac{1}{\operatorname{meas} \Gamma} \int_{\Gamma} \varphi(\mathbf{y}) \mathrm{d} \Gamma(\mathbf{y})
$$

will be used in the sequel.

Theorem 4. Let $\jmath_{1} \in \mathbf{H}(\operatorname{div}, \Omega)^{\prime}, \jmath_{2} \in L^{2}(\Omega)^{\prime}$ and $\jmath_{3} \in H_{0}^{1 / 2}(\Gamma)^{\prime}$. The following problem is well posed: find $\left(\mathbf{p}_{0}, u_{0}, \psi_{0}\right) \in \mathbf{H}(\operatorname{div}, \Omega) \times L^{2}(\Omega) \times H_{0}^{1 / 2}(\Gamma)$ such that

$$
\begin{array}{ll}
\left(\mathbf{p}_{0}, \mathbf{q}\right)_{\Omega}+\left(u_{0}, \operatorname{div} \mathbf{q}\right)_{\Omega}-\left\langle\gamma_{\nu} \mathbf{q}, \psi_{0}\right\rangle=\jmath_{1}(\mathbf{q}), & \forall \mathbf{q} \in \mathbf{H}(\operatorname{div}, \Omega), \\
\left(\operatorname{div} \mathbf{p}_{0}, v\right)_{\Omega}=\jmath_{2}(v), & \forall v \in L^{2}(\Omega), \\
\left\langle\left(\frac{1}{2} \mathrm{I}+\mathrm{K}\right)^{t} \gamma_{\nu} \mathbf{p}_{0}, \varphi\right\rangle+\left\langle\mathrm{W} \psi_{0}, \varphi\right\rangle=\jmath_{3}(\varphi), & \forall \varphi \in H_{0}^{1 / 2}(\Gamma),
\end{array}
$$


If the right-hand side is the same as (7), we obtain one of the solutions of (7). The solution of (11) can be recovered by:

$$
\mathbf{p}=\mathbf{p}_{0}, \quad u= \begin{cases}u_{0}+\mathrm{J}\left(\left(\frac{1}{2} I+\mathrm{K}\right) \psi_{0}-\mathrm{V}\left(\gamma_{\nu} \mathbf{p}_{0}-\beta_{1}\right)\right), & \text { in } \Omega, \\ \mathrm{D} \psi_{0}-\mathrm{S}\left(\gamma_{\nu} \mathbf{p}_{0}-\beta_{1}\right), & \text { in } \Omega^{e} .\end{cases}
$$

Note that the constant that corrects the interior solution in (13) can also be written as

$$
\frac{\left\langle\left(\frac{1}{2} \mathrm{I}+\mathrm{K}\right)^{t} 1, \psi_{0}\right\rangle-\left\langle\gamma_{\nu} \mathbf{p}_{0}-\beta_{1}, \mathrm{~V} 1\right\rangle}{\operatorname{meas} \Gamma} .
$$

Therefore, the integral operator $\mathrm{V}$ is only applied to the constant function and does not need to be discretized.

2.4. Galerkin discretization. We finally address the problem of the numerical approximation of the two well-posed problems above, i.e., (10) and (12). Since apart from some row changes, these problems are mutually transposed, we only need to study the discretization of one of them. For simplicity of the analysis, we choose to deal with (10). Take three finite dimensional spaces

$$
\mathbf{H}_{h} \subset \mathbf{H}(\operatorname{div}, \Omega), \quad L_{h} \subset L^{2}(\Omega), \quad X_{h} \subset H_{0}^{1 / 2}(\Gamma) .
$$

We want to compare the exact solution of (10) with its Galerkin discretization: find $\left(\mathbf{p}_{h}, u_{h}, \xi_{h}\right) \in \mathbf{H}_{h} \times L_{h} \times X_{h}$ such that

$$
\begin{array}{ll}
\left(\mathbf{p}_{h}, \mathbf{q}_{h}\right)_{\Omega}+\left(u_{h}, \operatorname{div} \mathbf{q}_{h}\right)_{\Omega}-\left\langle\gamma_{\nu} \mathbf{q}_{h},\left(\frac{1}{2} \mathrm{I}+\mathrm{K}\right) \xi_{h}\right\rangle=\ell_{1}\left(\mathbf{q}_{h}\right), & \forall \mathbf{q}_{h} \in \mathbf{H}_{h}, \\
\left(\operatorname{div} \mathbf{p}_{h}, v_{h}\right)_{\Omega}=\ell_{2}\left(v_{h}\right), & \forall v_{h} \in L_{h}, \\
\left\langle\gamma_{\nu} \mathbf{p}_{h}, \varphi\right\rangle+\left\langle\mathrm{W} \xi_{h}, \varphi_{h}\right\rangle=\ell_{3}\left(\varphi_{h}\right), & \forall \varphi_{h} \in X_{h} .
\end{array}
$$

The main result of this paper is the following stability result for the Galerkin method (16). Stability will be stated in the norms of $\mathbf{H}(\operatorname{div}, \Omega), L^{2}(\Omega)$ and $H^{1 / 2}(\Gamma)$, respectively, denoted

$$
\|\cdot\|_{\operatorname{div}, \Omega}, \quad\|\cdot\|_{\Omega}, \quad\|\cdot\|_{1 / 2, \Gamma} .
$$

Theorem 5. Assume that

$$
\operatorname{div} \mathbf{q}_{h} \in L_{h}, \quad \forall \mathbf{q}_{h} \in \mathbf{H}_{h}
$$

and that there exists $C_{0}>0$ such that

$$
\sup _{\mathbf{0} \neq \mathbf{q}_{h} \in \mathbf{H}_{h}} \frac{\left(\operatorname{div} \mathbf{q}_{h}, v_{h}\right)_{\Omega}}{\left\|\mathbf{q}_{h}\right\|_{\operatorname{div}, \Omega}} \geq C_{0}\left\|v_{h}\right\|_{\Omega}, \quad \forall v_{h} \in L_{h} .
$$

Then the Galerkin equations (16) are uniquely solvable. Moreover, there exists $C>0$ which depends on the discrete spaces only through the constant $C_{0}$ such that if $(\mathbf{p}, u, \xi)$ is the solution of (10), then the following quasi-optimality estimate holds:

$$
\begin{aligned}
& \left\|\mathbf{p}-\mathbf{p}_{h}\right\|_{\operatorname{div}, \Omega}+\left\|u-u_{h}\right\|_{\Omega}+\left\|\xi-\xi_{h}\right\|_{1 / 2, \Gamma} \\
& \quad \leq C\left(\inf _{\mathbf{q}_{h} \in \mathbf{H}_{h}}\left\|\mathbf{p}-\mathbf{q}_{h}\right\|_{\operatorname{div}, \Omega}+\inf _{v_{h} \in L_{h}}\left\|u-v_{h}\right\|_{\Omega}+\inf _{\varphi_{h} \in X_{h}}\left\|\xi-\varphi_{h}\right\|_{1 / 2, \Gamma}\right) .
\end{aligned}
$$




\section{AnAlysis of the CONTINUOUS PROBlEMS}

This section includes the detailed proof of Theorems [1, 2, 3] and 4, Although stated separately, these four results are very intimately connected and their proofs will follow as the consequence of a series of short propositions that will prove pieces of each until the moment when we can collect all of them.

Proposition 6. The set of solutions of (7) with a homogeneous right-hand side is the span of $(\mathbf{0}, 1,1)$.

Proof. First, note that

$$
\operatorname{ker} \mathrm{W}=\operatorname{ker}\left(\frac{1}{2} \mathrm{I}+\mathrm{K}\right)=\left\{\xi \in H^{1 / 2}(\Gamma): \mathrm{D} \xi \equiv 0 \quad \text { in } \Omega^{e}\right\}=\mathbb{P}_{0} .
$$

Then the triple $(\mathbf{0}, 1,1)$ solves (7) with homogeneous right-hand side. Reciprocally, elementary arguments show that a homogeneous solution of this problem satisfies

$$
\nabla u=\mathbf{p} \quad \text { in } \Omega, \quad \Delta u=0 \quad \text { in } \Omega, \quad \gamma u=\psi .
$$

Then $\left(\psi, \gamma_{\boldsymbol{\nu}} \mathbf{p}\right)$ are the Cauchy data of an interior solution of the Laplace equation in $\Omega$. Extending $u$ by zero in the exterior domain and using (2), we prove that

$$
\mathrm{S} \gamma_{\nu} \mathbf{p}-\mathrm{D} \psi=0, \quad \text { in } \Omega^{e}
$$

Taking the exterior normal derivative, it follows that

$$
\left(-\frac{1}{2} \mathrm{I}+\mathrm{K}\right)^{t} \gamma_{\boldsymbol{\nu}} \mathbf{p}+\mathrm{W} \psi=0
$$

This function is equal to $-\gamma_{\nu} \mathbf{p}$ by the third equation of (7) with zero on the righthand side. Hence $-\Delta u=0$ in $\Omega$ and $\partial_{\boldsymbol{\nu}} u=\gamma_{\boldsymbol{\nu}} \mathbf{p}=0$. Therefore, $u \equiv c$ for some $c \in \mathbb{P}_{0}$. By (20), it follows that $\mathbf{p}=\mathbf{0}$ and $\psi=c$.

Proposition 7. The set of solutions of (9) with a homogeneous right-hand side is the span of $(\mathbf{0}, 0,1)$.

Proof. Property (19) proves that $(\mathbf{0}, 0,1)$ is a homogeneous solution of (9). If, on the other hand, we have a solution $(\mathbf{p}, u, \xi)$ of (9) with a homogeneous right-hand side, then

$$
\nabla u=\mathbf{p} \quad \text { in } \Omega, \quad \Delta u=0 \quad \text { in } \Omega, \quad \gamma u=\left(\frac{1}{2} \mathrm{I}+\mathrm{K}\right) \xi .
$$

Now extend $u$ by $u:=\mathrm{D} \xi$ in $\Omega^{e}$. Then

$$
\gamma^{e} u=\left(\frac{1}{2} \mathrm{I}+\mathrm{K}\right) \xi=\gamma u, \quad \partial_{\nu}^{e} u=-\mathrm{W} \xi=\gamma_{\boldsymbol{\nu}} \mathbf{p}=\partial_{\boldsymbol{\nu}} u
$$

Therefore, $(\mathbf{p}, u)$ is a solution of the transmission problem (11) with homogeneous data. Uniqueness of the solution of (1) implies that $u=0\left(\right.$ in $\left.\Omega \cup \Omega^{e}\right)$ and $\mathbf{p}=0$. Since $\mathrm{D} \xi=0$ in $\Omega^{e}$, by (19) we prove that $\xi \in \mathbb{P}_{0}$.

Proposition 8. The operator associated to problem (9) is Fredholm of index zero.

Proof. Take $\widetilde{\Phi}:=\exp (-r) /(4 \pi r)$, which is the fundamental solution of the elliptic operator $u \mapsto-\Delta u+u$. Then define $\widetilde{\mathrm{K}}$ and $\widetilde{\mathrm{W}}$ with this new fundamental solution using (4) and (5). We now consider the problem: find $(\mathbf{p}, \xi, u) \in \mathbf{H}(\operatorname{div}, \Omega) \times$ $H^{1 / 2}(\Gamma) \times L^{2}(\Omega)$ such that

$$
\begin{array}{ll}
(\mathbf{p}, \mathbf{q})_{\Omega}-\left\langle\gamma_{\nu} \mathbf{q},\left(\frac{1}{2} \mathrm{I}+\widetilde{\mathrm{K}}\right) \xi\right\rangle+(u, \operatorname{div} \mathbf{q})_{\Omega}=\ell_{1}(\mathbf{q}), & \forall \mathbf{q} \in \mathbf{H}(\operatorname{div}, \Omega), \\
\left\langle\gamma_{\nu} \mathbf{p}, \varphi\right\rangle+\langle\widetilde{\mathrm{W}} \xi, \varphi\rangle=\ell_{2}(\varphi), & \forall \varphi \in H^{1 / 2}(\Gamma), \\
(\operatorname{div} \mathbf{p}, v)_{\Omega}=\ell_{3}(v), & \forall v \in L^{2}(\Omega),
\end{array}
$$


for an arbitrary right-hand side in the dual space. Because $\mathrm{W}-\widetilde{\mathrm{W}}$ and $\mathrm{K}-\widetilde{\mathrm{K}}$ are compact, once we have proved that the operator associated to problem (22) is invertible, we will obtain as a consequence that the operator associated to (9) is Fredholm of index zero.

To prove that problem (22) is well posed we will use the general theory of mixed problems [3, Chapter II]. First, $\operatorname{div}: \mathbf{H}(\operatorname{div}, \Omega) \rightarrow L^{2}(\Omega)$ is surjective so we only have to concentrate on proving well posedness of the following problems: find $(\mathbf{p}, \xi) \in \mathbf{V} \times H^{1 / 2}(\Gamma)$ such that

$$
\begin{array}{ll}
(\mathbf{p}, \mathbf{q})_{\Omega}-\left\langle\gamma_{\nu} \mathbf{q},\left(\frac{1}{2} \mathrm{I}+\widetilde{\mathrm{K}}\right) \xi\right\rangle=\jmath(\mathbf{q}), & \forall \mathbf{q} \in \mathbf{V}, \\
\left\langle\gamma_{\nu} \mathbf{p}, \varphi\right\rangle+\langle\widetilde{\mathrm{W}} \xi, \varphi\rangle=\langle\mu, \varphi\rangle, & \forall \varphi \in H^{1 / 2}(\Gamma),
\end{array}
$$

where

$$
\mathbf{V}:=\{\mathbf{q} \in \mathbf{H}(\operatorname{div}, \Omega): \operatorname{div} \mathbf{q}=0\}=\left\{\mathbf{q} \in\left(L^{2}(\Omega)\right)^{3}: \operatorname{div} \mathbf{q}=0\right\}
$$

and $(\jmath, \mu) \in \mathbf{V}^{\prime} \times H^{-1 / 2}(\Gamma)$ is an arbitrary right-hand side. The solution of this problem is given by $\xi=\widetilde{\mathrm{W}}^{-1}\left(\mu-\gamma_{\boldsymbol{\nu}} \mathbf{p}\right)$ and $\mathbf{p} \in \mathbf{V}$ satisfying

$$
(\mathbf{p}, \mathbf{q})_{\Omega}-\left\langle\gamma_{\nu} \mathbf{q},\left(\frac{1}{2} \mathrm{I}+\widetilde{\mathrm{K}}\right) \widetilde{\mathrm{W}}^{-1} \gamma_{\nu} \mathbf{p}\right\rangle=\jmath(\mathbf{q})-\left\langle\gamma_{\nu} \mathbf{q},\left(\frac{1}{2} \mathrm{I}+\widetilde{\mathrm{K}}\right) \widetilde{\mathrm{W}}^{-1} \mu\right\rangle, \quad \forall \mathbf{q} \in \mathbf{V}
$$

The operator $-\left(\frac{1}{2} \mathrm{I}+\widetilde{\mathrm{K}}\right) \widetilde{\mathrm{W}}^{-1}$ is an integral representation of the Neumann-toDirichlet operator for the equation $-\Delta u+u=0$ in $\Omega^{e}$, which is elliptic. Therefore, the bilinear form $-\left\langle\gamma_{\nu} \mathbf{q},\left(\frac{1}{2} \mathrm{I}+\widetilde{\mathrm{K}}\right) \widetilde{\mathrm{W}}^{-1} \gamma_{\boldsymbol{\nu}} \mathbf{p}\right\rangle$ is nonnegative and problem (25) is elliptic. This proves the result.

Proposition 9. Equations (7) are solvable for the given right-hand side.

Proof. By a transposition argument, Proposition 8 also proves that the operator associated to (7) is Fredholm of index zero. Therefore, the problem is solvable if and only if the right-hand side vanishes when applied to any element of the kernel of the transposed problem which is $\operatorname{span}\{(\mathbf{0}, 0,1)\}$ (see Proposition 7). However, by (19)

$$
\left\langle\left(\frac{1}{2} \mathrm{I}+\mathrm{K}\right)^{t} \beta_{1}, 1\right\rangle=\left\langle\beta_{1},\left(\frac{1}{2} \mathrm{I}+\mathrm{K}\right) 1\right\rangle=0,
$$

and then the condition is satisfied.

Proposition 10. Equations (9) are solvable for the given right-hand side if and only if (8) holds.

Proof. The operator in (9) is Fredholm of index zero and the kernel of its transpose is $\operatorname{span}\{(\mathbf{0}, 1,-1)\}$. This is proved in Proposition 6 above, up to a change of sign needed to identify the transpose of (9) with (7). Condition (8) is just the solvability condition for this problem applied to the particular right-hand side of (9).

Proposition 11. Problem (10) is well posed.

Proof. Note that we will give an alternative proof of this result as a byproduct of Section 4 (Proposition 17). Let us consider problem (9) with the following righthand side:

$$
\jmath_{1}(\mathbf{q}):=\ell_{1}(\mathbf{q}), \quad \jmath_{2}(v):=\ell_{2}(v), \quad \jmath_{3}(\varphi):=\ell_{3}(\varphi-\mathrm{J} \varphi)+\mathrm{J} \varphi \ell_{2}(1) .
$$

This right-hand side satisfies the solvability condition for problem (9) which is $\jmath_{2}(1)-\jmath_{3}(1)=0$. Solutions of (9) are defined modulo an additive constant in the third component. Therefore, only one of them solves (10). 
Proposition 12. Problem (12) is well posed.

Proof. This follows from a simple transposition argument from Proposition 11,

Remark. Theorems 1, 2 and 3 are proven by Propositions 6 to 11

Proof of Theorem 4. It remains to show how to obtain a solution of the transmission problem (11) from the unique solution of (12) with the right-hand side of (7). By (19), the solution of (12) solves (7) too. Therefore,

$$
\nabla u_{0}=\mathbf{p}_{0} \quad \text { in } \Omega, \quad-\Delta u_{0}=f \quad \text { in } \Omega, \quad \gamma u_{0}=\psi_{0}+\beta_{0} .
$$

We extend $u_{0}$ to

$$
u_{0}:=\mathrm{D} \psi_{0}-\mathrm{S}\left(\partial_{\boldsymbol{\nu}} u_{0}-\beta_{1}\right), \quad \text { in } \Omega^{e} .
$$

Using the third equation of (77) and the jump relations, it follows that $\partial_{\nu}^{e} u_{0}=$ $\partial_{\nu} u_{0}-\beta_{1}$. Therefore, we can compare the exterior definition of $u_{0}$ with the integral representation given by Green's Third Identity

$$
\mathrm{D} \psi_{0}-\mathrm{S} \partial_{\nu}^{e} u_{0}=\mathrm{D} \gamma^{e} u_{0}-\mathrm{S} \partial_{\nu}^{e} u_{0} .
$$

Therefore, by (19), there exists $c$ such that $\psi_{0}+c=\gamma^{e} u_{0}$ and this constant can be computed comparing the averages of both sides of this last equality

$$
c=\mathrm{J} \gamma^{e} u_{0}=\mathrm{J} \gamma^{e}\left(\mathrm{D} \psi_{0}-\mathrm{S}\left(\gamma_{\nu} \mathbf{p}_{0}-\beta_{1}\right)\right) .
$$

It is clear that adding this constant to the interior value of $u_{0}$ and to $\psi_{0}$ we obtain the solution of (11). The jump relations show that the constant (26) is just the correction given in (13).

\section{Analysis of the Discrete PRoblems}

The aim of this section is to prove Theorem 5 Actually, we will prove a more general result (Proposition 17), from which Theorem 5 will be a consequence. Throughout this section we are given three closed spaces (15) satisfying (17) and (18). We will not need them to be finite dimensional. In particular, they can be taken to be the full spaces. Note that (17) implies that

$$
\begin{aligned}
\mathbf{V}_{h} & :=\left\{\mathbf{q}_{h} \in \mathbf{H}_{h}:\left(\operatorname{div} \mathbf{q}_{h}, v_{h}\right)_{\Omega}=0, \quad \forall v_{h} \in L_{h}\right\} \\
& =\left\{\mathbf{q}_{h} \in \mathbf{H}_{h}: \operatorname{div} \mathbf{q}_{h}=0\right\} \subset \mathbf{V},
\end{aligned}
$$

where $\mathbf{V}$ is given by (24). We will use a general result on the invertibility of problems with mixed form to work out an analysis eliminating $u_{h}$ as an unknown.

Let us first concentrate our efforts in the following reduced problem. We are given closed subspaces $\mathbf{V}_{h} \subset \mathbf{V}$ and $X_{h} \subset H_{0}^{1 / 2}(\Gamma)$ and linear functionals $\ell_{1, h} \in \mathbf{V}_{h}^{\prime}$, $\ell_{2, h} \in X_{h}^{\prime}$. We then consider the problem: find $\left(\mathbf{p}_{h}, \xi_{h}\right) \in \mathbf{V}_{h} \times X_{h}$ such that

$$
\begin{array}{ll}
\left(\mathbf{p}_{h}, \mathbf{q}_{h}\right)_{\Omega}-\left\langle\gamma_{\boldsymbol{\nu}} \mathbf{q}_{h},\left(\frac{1}{2} \mathrm{I}+\mathrm{K}\right) \xi_{h}\right\rangle=\ell_{1, h}\left(\mathbf{q}_{h}\right), & \forall \mathbf{q}_{h} \in \mathbf{V}_{h}, \\
\left\langle\gamma_{\nu} \mathbf{p}_{h}, \varphi\right\rangle+\left\langle\mathrm{W} \xi_{h}, \varphi_{h}\right\rangle=\ell_{2, h}\left(\varphi_{h}\right), & \forall \varphi_{h} \in X_{h} .
\end{array}
$$

Note that (28) can be considered as a Galerkin discretization of the coupling of a dual and a boundary integral formulations for problem (1). This auxiliary problem is defined for analytical reasons, although it is an object of its own interest as a different coupling of BEM with divergence-free finite elements. 
Proposition 13. Let $\left(\mathbf{p}_{h}, \xi_{h}\right)$ be a solution of (28) and let $u^{*}:=\mathrm{D} \xi_{h}$. Then $\left(\mathbf{p}_{h}, u^{*}\right)$ is a solution of the following: find $\left(\mathbf{p}_{h}, u^{*}\right) \in \mathbf{V}_{h} \times W^{1}\left(\Omega \cup \Omega^{e}\right)$ such that

$$
\begin{array}{ll}
-\Delta u^{*}=0 \quad \text { in } \Omega \cup \Omega^{e}, & \\
{\left[\gamma u^{*}\right] \in X_{h},} & \\
{\left[\partial_{\boldsymbol{\nu}} u^{*}\right]=0,} & \\
\left(\mathbf{p}_{h}, \mathbf{q}_{h}\right)_{\Omega}-\left\langle\gamma_{\nu} \mathbf{q}_{h}, \gamma^{e} u^{*}\right\rangle=\ell_{1, h}\left(\mathbf{q}_{h}\right), & \forall \mathbf{q}_{h} \in \mathbf{V}_{h}, \\
\left\langle\gamma_{\nu} \mathbf{p}_{h}-\partial_{\boldsymbol{\nu}}^{e} u^{*}, \varphi_{h}\right\rangle=\ell_{2, h}\left(\varphi_{h}\right), & \forall \varphi_{h} \in X_{h} .
\end{array}
$$

Reciprocally, given a solution of (29), then $\left(\mathbf{p}_{h},-\left[\gamma u^{*}\right]\right)$ is a solution of (28).

Proof. It is a direct consequence of the jump conditions of potentials and of Green's Third Identity (2).

Consider now the space

$$
W:=\left\{u^{*} \in W^{1}\left(\Omega \cup \Omega^{e}\right):\left[\gamma u^{*}\right] \in H_{0}^{1 / 2}(\Gamma)\right\}
$$

and its subspace

$$
W_{h}:=\left\{u^{*} \in W^{1}\left(\Omega \cup \Omega^{e}\right):\left[\gamma u^{*}\right] \in X_{h}\right\} .
$$

An argument in [27, Section 4] shows that the seminorm $\left\|\nabla u^{*}\right\|_{\Omega \cup \Omega^{e}}$ is a norm in $W$ equivalent to the usual one.

Proposition 14. Problem (29) is equivalent to: find $\left(\mathbf{p}_{h}, u^{*}\right) \in \mathbf{V}_{h} \times W_{h}$ such that

$$
\begin{array}{ll}
\left(\mathbf{p}_{h}, \mathbf{q}_{h}\right)_{\Omega}-\left\langle\gamma_{\nu} \mathbf{q}_{h}, \gamma^{e} u^{*}\right\rangle=\ell_{1, h}\left(\mathbf{q}_{h}\right), & \forall \mathbf{q}_{h} \in \mathbf{V}_{h}, \\
\left\langle\gamma_{\nu} \mathbf{p}_{h},\left[\gamma v^{*}\right]\right\rangle-\left(\nabla u^{*}, \nabla v^{*}\right)_{\Omega \cup \Omega^{e}}=\ell_{2, h}\left(\left[\gamma v^{*}\right]\right), & \forall v^{*} \in W_{h} .
\end{array}
$$

Proof. Let $\left(\mathbf{p}_{h}, u^{*}\right)$ be a solution of (30). Testing the second equation with a general $\mathcal{C}^{\infty}$ function, compactly supported in $\Omega \cup \Omega^{e}$, we prove that $\Delta u^{*}=0$ in $\Omega \cup \Omega^{e}$. The second equation of (30) can then be rewritten as

$$
\left\langle\gamma_{\nu} \mathbf{p}_{h},\left[\gamma v^{*}\right]\right\rangle-\left\langle\partial_{\nu} u^{*}, \gamma v^{*}\right\rangle+\left\langle\partial_{\nu}^{e} u^{*}, \gamma^{e} v^{*}\right\rangle=\ell_{2, h}\left(\left[\gamma v^{*}\right]\right), \quad \forall v^{*} \in W_{h} .
$$

Given $\varphi \in H^{1 / 2}(\Gamma)$ we can construct $v^{*} \in H^{1}\left(\mathbb{R}^{3}\right) \subset W^{1}\left(\mathbb{R}^{3}\right) \subset W_{h}$ with $\gamma v^{*}=$ $\gamma^{e} v^{*}=\varphi$. Testing (31) with this $v^{*}$ we prove that

$$
\left\langle\left[\partial_{\nu} u^{*}\right], \varphi\right\rangle=0, \quad \forall \varphi \in H^{1 / 2}(\Gamma),
$$

that is, $\left[\partial_{\nu} u^{*}\right]=0$. Plugging this new condition into (31) we obtain

$$
\left\langle\gamma_{\nu} \mathbf{p}_{h}-\partial_{\boldsymbol{\nu}} u^{*},\left[\gamma v^{*}\right]\right\rangle=\ell_{2, h}\left(\left[\gamma v^{*}\right]\right), \quad \forall v^{*} \in W_{h} .
$$

Finally, given $\varphi_{h} \in X_{h}$, we can construct $v^{*} \in H^{1}(\Omega)$ such that $\gamma v^{*}=\varphi_{h}$ and extend it by zero to $\Omega^{e}$. This function is in $W_{h}$ and shows that (32) is equivalent to the last equation of (29).

Reciprocally, if we have a solution of (29), then (32) is satisfied. Therefore, so is (31) because $\left[\partial_{\nu} u^{*}\right]=0$. Finally, (31) and $\Delta u^{*}=0$ in $\Omega \cup \Omega^{e}$ imply the second equation of (30).

Proposition 15. The following bilinear form in $\mathbf{V} \times W$ is continuous and elliptic:

$$
a\left(\left(\mathbf{p}, u^{*}\right),\left(\mathbf{q}, v^{*}\right)\right)=(\mathbf{p}, \mathbf{q})_{\Omega}-\left\langle\gamma_{\boldsymbol{\nu}} \mathbf{q}, \gamma^{e} u^{*}\right\rangle-\left\langle\gamma_{\boldsymbol{\nu}} \mathbf{p},\left[\gamma v^{*}\right]\right\rangle+\left(\nabla u^{*}, \nabla v^{*}\right)_{\Omega \cup \Omega^{e}} .
$$


Proof. Continuity of the bilinear form is straightforward. Recalling that elements of $\mathbf{V}$ are divergence free, it follows that

$$
\begin{aligned}
a\left(\left(\mathbf{p}, u^{*}\right),\left(\mathbf{p}, u^{*}\right)\right) & =(\mathbf{p}, \mathbf{p})_{\Omega}-\left\langle\gamma_{\nu} \mathbf{p}, \gamma u^{*}\right\rangle+\left(\nabla u^{*}, \nabla u^{*}\right)_{\Omega \cup \Omega^{e}} \\
& =(\mathbf{p}, \mathbf{p})_{\Omega}-\left(\mathbf{p}, \nabla u^{*}\right)_{\Omega}+\left(\nabla u^{*}, \nabla u^{*}\right)_{\Omega \cup \Omega^{e}} \\
& \geq \frac{1}{2}\|\mathbf{p}\|_{\Omega}^{2}+\frac{1}{2}\left\|\nabla u^{*}\right\|_{\Omega \cup \Omega^{e}}^{2} .
\end{aligned}
$$

Since the Sobolev seminorm is equivalent to the usual norm in $W$ and the $L^{2}$ norm is equivalent to the $\mathbf{H}(\operatorname{div}, \Omega)$ norm in $\mathbf{V}$, the result is proven.

Proposition 16. Problem (28) has a unique solution. Moreover, there exists a positive constant $C$ independent of the particular choice of the spaces $\mathbf{V}_{h}$ and $X_{h}$ such that

$$
\left\|\mathbf{p}_{h}\right\|_{\mathrm{div}, \Omega}+\left\|\xi_{h}\right\|_{1 / 2, \Gamma} \leq C\left(\left\|\ell_{1, h}\right\|_{\mathbf{V}_{h}^{\prime}}+\left\|\ell_{2, h}\right\|_{X_{h}^{\prime}}\right) .
$$

Proof. Existence and uniqueness of the solution of (28) follows from Propositions 13. 14 and 15. The ellipticity of the bilinear form (Proposition 15) allows us to bound the $\mathbf{H}(\operatorname{div}, \Omega) \times W^{1}\left(\Omega \cup \Omega^{e}\right)$ norm of $\left(\mathbf{p}_{h}, u^{*}\right)$ uniformly in terms of the norm of the right-hand side of (28). Finally, $\xi_{h}=\left[\gamma u^{*}\right]$, which permits us to bound the $H^{1 / 2}(\Gamma)$ norm of this unknown of the system.

Proposition 17. Let $\mathbf{H}_{h}, L_{h}$ and $X_{h}$ be closed subspaces (15) satisfying (17) and (18). Then for all $\ell_{1, h} \in \mathbf{H}_{h}^{\prime}, \ell_{2, h} \in X_{h}^{\prime}, \ell_{3, h} \in L_{h}^{\prime}$, the following problem admits a unique solution: find $\left(\mathbf{p}_{h}, \xi_{h}, u_{h}\right) \in \mathbf{H}_{h} \times X_{h} \times L_{h}$ such that

$$
\begin{array}{ll}
\left(\mathbf{p}_{h}, \mathbf{q}_{h}\right)_{\Omega}-\left\langle\gamma_{\nu} \mathbf{q}_{h},\left(\frac{1}{2} \mathrm{I}+\mathrm{K}\right) \xi_{h}\right\rangle+\left(u_{h}, \operatorname{div} \mathbf{q}_{h}\right)_{\Omega}=\ell_{1, h}\left(\mathbf{q}_{h}\right), & \forall \mathbf{q}_{h} \in \mathbf{H}_{h}, \\
\left\langle\gamma_{\nu} \mathbf{p}_{h}, \varphi\right\rangle+\left\langle\mathrm{W} \xi_{h}, \varphi_{h}\right\rangle=\ell_{2, h}\left(\varphi_{h}\right), & \forall \varphi_{h} \in X_{h}, \\
\left(\operatorname{div} \mathbf{p}_{h}, v_{h}\right)_{\Omega}=\ell_{3, h}\left(v_{h}\right), & \forall v_{h} \in L_{h} .
\end{array}
$$

Moreover, there exists $C>0$ which depends only on $C_{0}$ in (18) and is otherwise independent of the choice of the spaces, such that

$$
\left\|\mathbf{p}_{h}\right\|_{\operatorname{div}, \Omega}+\left\|\xi_{h}\right\|_{1 / 2, \Gamma}+\left\|u_{h}\right\|_{\Omega} \leq C\left(\left\|\ell_{1, h}\right\|_{\mathbf{H}_{h}^{\prime}}+\left\|\ell_{2, h}\right\|_{X_{h}^{\prime}}+\left\|\ell_{3, h}\right\|_{L_{h}^{\prime}}\right) .
$$

Proof. The result follows from a straightforward application of general results on problems with mixed form. Note that we have reordered equations and unknowns to emphasize the mixed structure of the problem, with $\mathbf{H}_{h} \times X_{h}$ as a space for primal unknowns and $L_{h}$ as a space for 'Lagrange multipliers'.

Because of (17), the discrete kernel $\mathbf{V}_{h} \times X_{h}$ is a subspace of $\mathbf{V} \times H_{0}^{1 / 2}(\Gamma)$ (see (27)) and then existence and uniqueness of the solution are equivalent to: (a) an inf-sup condition (18); (b) an invertibility property for variational problems set in $\mathbf{V}_{h} \times X_{h}$ (Proposition 16). The norm of the operator that assigns solutions of (33) to right-hand sides depends on the (inverse of the) constant $C_{0}$ in the infsup condition (18) and on the norm of the inversion operator for equations (28). As proven in Proposition 16, this last constant does not depend on the particular choice of spaces.

Proof of Theorem 5. For Galerkin approximation of well-posed problems, the quasioptimality or Céa estimate is equivalent to stability. This means that the estimate in the statement of Theorem 5 is a consequence of the uniform invertibility estimate (34) for problem (33). The proportionality constant between the Céa estimate and the uniform bound of the inverse depends only on the norm of the operator 
and therefore both are independent of the choice of discrete spaces (apart from dependence through $C_{0}$ in the inf-sup condition).

\section{Neumann Boundary CONDItions}

In this section we change the transmission problem to one that takes place in the exterior of a bounded domain $\Omega_{\text {obs }}$ with Lipschitz boundary $\Sigma$. Surrounding this domain there is another $\mathrm{O}$. The union of both and their common interface corresponds to our original domain $\Omega$. The exterior domain will still be referred to as $\Omega^{e}$ and $\Gamma$ will be the interface separating $\Omega$ from $\Omega^{e}$. A sketch of this new geometric setting is given in Figure 1. The superscript $\Sigma$ will be used for traces and normal derivatives on $\Sigma$.

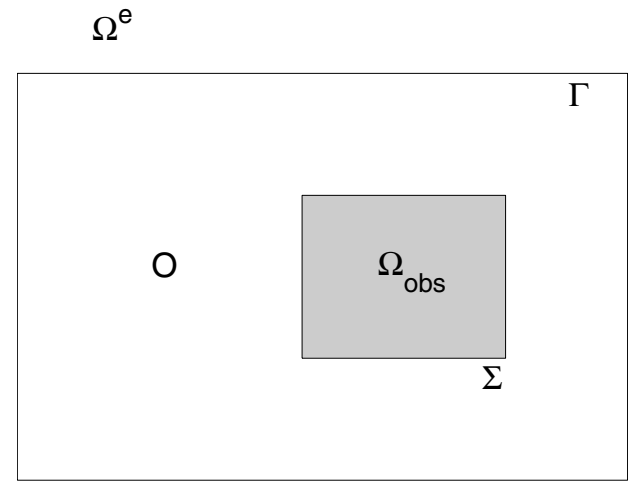

Figure 1. Geometric setting for Sections 5 and 6

We consider the new transmission problem

$$
\begin{array}{ll}
\gamma_{\boldsymbol{\nu}}^{\Sigma} \mathbf{p}=\beta_{\mathrm{Neu}}, & \\
\mathbf{p}-\nabla u=0 \quad \text { in } \mathrm{O}, & -\operatorname{div} \mathbf{p}=f \quad \text { in } \mathrm{O}, \\
{[\gamma u]=\beta_{0},} & \gamma_{\boldsymbol{\nu}} \mathbf{p}=\partial_{\boldsymbol{\nu}}^{e} u+\beta_{1}, \\
-\Delta u=0 \quad \text { in } \Omega^{e}, & \\
u=\mathcal{O}\left(|\mathbf{x}|^{-1}\right) \text { as }|\mathbf{x}| \rightarrow \infty, &
\end{array}
$$

with $\beta_{\text {Neu }} \in H^{-1 / 2}(\Sigma)$. We will start with the indirect formulation, trying to write $u=\mathrm{D} \xi$ in $\Omega^{e}$. Following the argument given in Section 2, we show that a necessary condition for this is

$$
(f, 1)_{\mathrm{O}}+\left\langle\beta_{1}, 1\right\rangle+\left\langle\beta_{\mathrm{Neu}}, 1\right\rangle_{\Sigma}=0
$$

where $\langle\cdot, \cdot\rangle_{\Sigma}$ is the duality product between $H^{-1 / 2}(\Sigma)$ and $H^{1 / 2}(\Sigma)$. We will need the following space:

$$
\mathbf{H}_{\Sigma}(\operatorname{div}, \mathrm{O}):=\left\{\mathbf{q} \in \mathbf{H}(\operatorname{div}, \mathrm{O}): \gamma_{\boldsymbol{\nu}}^{\Sigma} \mathbf{q}=0\right\} .
$$


The first coupled formulation is: find $(\mathbf{p}, u, \xi) \in \mathbf{H}(\operatorname{div}, \mathrm{O}) \times L^{2}(\mathrm{O}) \times H^{1 / 2}(\Gamma)$ such that $\gamma_{\nu}^{\Sigma} \mathbf{p}=\beta_{\mathrm{Neu}}$ and

$$
\begin{array}{ll}
(\mathbf{p}, \mathbf{q})_{\mathrm{O}}+(u, \operatorname{div} \mathbf{q})_{\mathrm{O}}-\left\langle\gamma_{\boldsymbol{\nu}} \mathbf{q},\left(\frac{1}{2} \mathrm{I}+\mathrm{K}\right) \xi\right\rangle=\left\langle\gamma_{\boldsymbol{\nu}} \mathbf{q}, \beta_{0}\right\rangle, & \forall \mathbf{q} \in \mathbf{H}_{\Sigma}(\operatorname{div}, \mathrm{O}), \\
(\operatorname{div} \mathbf{p}, v)_{\mathrm{O}}=-(f, v)_{\mathrm{O}}, & \forall v \in L^{2}(\mathrm{O}), \\
\left\langle\gamma_{\boldsymbol{\nu}} \mathbf{p}, \varphi\right\rangle+\langle\mathrm{W} \xi, \varphi\rangle=\left\langle\beta_{1}, \varphi\right\rangle, & \forall \varphi \in H^{1 / 2}(\Gamma) .
\end{array}
$$

We will call reduced formulation to the modification of (37) by looking for $\xi$ in $H_{0}^{1 / 2}(\Gamma)$ and testing the third variational equation in this same space. A Galerkin method for the reduced formulation uses three spaces,

$$
\mathbf{H}_{h}^{\mathrm{O}} \subset \mathbf{H}(\operatorname{div}, \mathrm{O}), \quad L_{h}^{\mathrm{O}} \subset L^{2}(\mathrm{O}), \quad X_{h} \subset H_{0}^{1 / 2}(\Gamma),
$$

a suitable approximation of the essential boundary condition

$$
\beta_{\mathrm{Neu}} \approx \beta_{\mathrm{Neu}}^{h} \in \gamma_{\nu}^{\Sigma} \mathbf{H}_{h}^{\mathrm{O}}
$$

and the space $\mathbf{H}_{h, \Sigma}^{\mathrm{O}}:=\mathbf{H}_{h}^{\mathrm{O}} \cap \mathbf{H}_{\Sigma}(\operatorname{div}, \mathrm{O})$. The Galerkin equations are: find $\left(\mathbf{p}_{h}, u_{h}, \xi_{h}\right) \in \mathbf{H}_{h}^{\mathrm{O}} \times L_{h}^{\mathrm{O}} \times X_{h}$ such that $\gamma_{\nu}^{\Sigma} \mathbf{p}_{h}=\beta_{\mathrm{Neu}}^{h}$ and

$$
\begin{array}{ll}
\left(\mathbf{p}_{h}, \mathbf{q}_{h}\right)_{\mathrm{O}}+\left(u_{h}, \operatorname{div} \mathbf{q}_{h}\right)_{\mathrm{O}}-\left\langle\gamma_{\boldsymbol{\nu}} \mathbf{q}_{h},\left(\frac{1}{2} \mathrm{I}+\mathrm{K}\right) \xi_{h}\right\rangle & =\left\langle\gamma_{\boldsymbol{\nu}} \mathbf{q}_{h}, \beta_{0}\right\rangle, \\
\left(\operatorname{div} \mathbf{p}_{h}, v_{h}\right)_{\mathrm{O}}=-\left(f, v_{h}\right)_{\mathrm{O}}, & \forall \mathbf{q}_{h} \in \mathbf{H}_{h, \Sigma}^{\mathrm{O}}, \\
\left\langle\gamma_{\boldsymbol{\nu}} \mathbf{p}_{h}, \varphi_{h}\right\rangle+\left\langle\mathrm{W} \xi_{h}, \varphi_{h}\right\rangle=\left\langle\beta_{1}, \varphi_{h}\right\rangle, & \forall v_{h} \in L_{h}^{\mathrm{O}}, \\
& \forall \varphi_{h} \in X_{h} .
\end{array}
$$

Theorem 18. Problem (37) is solvable, up to an additive constant in $\xi$ if and only if (36) holds. Its reduced formulation is well posed. The Galerkin equations (38) are uniquely solvable if the following discrete conditions are met:

$$
\operatorname{div} \mathbf{H}_{h, \Sigma}^{\mathrm{O}} \subset L_{h}^{\mathrm{O}}, \quad \sup _{0 \neq \mathbf{q}_{h} \in \mathbf{H}_{h, \Sigma}^{\mathrm{O}}} \frac{\left(\operatorname{div} \mathbf{q}_{h}, v_{h}\right)_{\mathrm{O}}}{\left\|\mathbf{q}_{h}\right\|_{\operatorname{div}, \mathrm{O}}} \geq C_{0}\left\|v_{h}\right\|_{\mathrm{O}}, \quad \forall v_{h} \in L_{h}^{\mathrm{O}} .
$$

Finally, the following quasi-optimality estimate holds:

$$
\begin{aligned}
&\left\|\mathbf{p}-\mathbf{p}_{h}\right\|_{\text {div }, \mathrm{O}}+\| u-u_{h}\left\|_{\mathrm{O}}+\right\| \xi-\xi_{h} \|_{1 / 2, \Gamma} \\
& \leq C( \inf \left\{\left\|\mathbf{p}-\mathbf{q}_{h}\right\|_{\text {div }, \mathrm{O}}: \mathbf{q}_{h} \in \mathbf{H}_{h}^{\mathrm{O}}, \quad \gamma_{\boldsymbol{\nu}} \mathbf{q}_{h}=\beta_{\text {Neu }}^{h}\right\} \\
&\left.\quad+\inf _{v_{h} \in L_{h}^{\mathrm{O}}}\left\|u-v_{h}\right\|_{\mathrm{O}}+\inf _{\varphi_{h} \in X_{h}}\left\|\xi-\varphi_{h}\right\|_{1 / 2, \Gamma}\right) .
\end{aligned}
$$

The constant $C>0$ depends on $C_{0}$ but is otherwise independent of the choice of spaces.

Proof. One of the interesting byproducts of the way we have developed the analysis in Section 4 is the fact that most results in this section are just a particular case of the ones of the problem set in free space.

Problem (37) can be rewritten in the variable $\mathbf{p}_{0}:=\mathbf{p}-\mathbf{p}_{\mathrm{Neu}} \in \mathbf{H}_{\Sigma}(\operatorname{div}, \mathrm{O})$, where $\gamma_{\nu}^{\Sigma} \mathbf{p}_{\mathrm{Neu}}=\beta_{\mathrm{Neu}}$. The right-hand side is given by

$$
\begin{aligned}
\ell_{1}(\mathbf{q}) & :=\left\langle\gamma_{\boldsymbol{\nu}} \mathbf{q}, \beta_{0}\right\rangle-\left(\mathbf{p}_{\mathrm{Neu}}, \mathbf{q}\right)_{\mathrm{O}}, \\
\ell_{2}(v) & :=-(f, v)_{\mathrm{O}}-\left(\operatorname{div} \mathbf{p}_{\mathrm{Neu}}, v\right)_{\mathrm{O}}, \\
\ell_{3}(\varphi) & :=\left\langle\beta_{1}-\gamma_{\boldsymbol{\nu}} \mathbf{p}_{\mathrm{Neu}}, \varphi\right\rangle .
\end{aligned}
$$

The theory for problem (9) (that is, Theorem 24) can be repeated for this new problem. The kernel of the associated operator is the span of $(\mathbf{0}, 0,1)$ and solvability 
is subject to cancelation of the right-hand side when applied to $(\mathbf{0}, 1,-1)$, which spans the kernel of the transpose. This compatibility condition reads

$$
(f, 1)_{\mathrm{O}}+\left(\operatorname{div} \mathbf{p}_{\mathrm{Neu}}, 1\right)_{\mathrm{O}}+\left\langle\beta_{1}, 1\right\rangle-\left\langle\gamma_{\boldsymbol{\nu}} \mathbf{p}_{\mathrm{Neu}}, 1\right\rangle=0,
$$

which is easily shown to be equivalent to (36) thanks to the condition $\gamma_{\nu}^{\Sigma} \mathbf{p}_{\mathrm{Neu}}=$ $\beta_{\mathrm{Neu}}$.

Let us now consider the reduced problem and its Galerkin discretization. Note first that

$$
\mathbf{H}_{\Sigma}(\operatorname{div}, \mathrm{O}) \cong \mathbf{H}_{0}:=\left\{\mathbf{q} \in \mathbf{H}(\operatorname{div}, \Omega): \mathbf{q} \equiv 0 \quad \text { in } \Omega_{\mathrm{obs}}\right\} \subset \mathbf{H}(\operatorname{div}, \Omega)
$$

and

$$
L^{2}(\mathrm{O}) \cong L_{0}:=\left\{v \in L^{2}(\Omega): v \equiv 0 \quad \text { in } \Omega_{\mathrm{obs}}\right\} .
$$

The reduced formulation can then be written as a particular case of problem (33) with $\mathbf{H}_{h}=\mathbf{H}_{0}, L_{h}=L_{0}, X_{h}=H_{0}^{1 / 2}(\Gamma)$. A direct application of Proposition 16 then gives the well posedness of the reduced problem.

After having chosen $\mathbf{p}_{\mathrm{Neu}}^{h} \in \mathbf{H}_{h}^{\mathrm{O}}$ such that $\gamma_{\boldsymbol{\nu}}^{\Sigma} \mathbf{p}_{\mathrm{Neu}}^{h}=\beta_{\mathrm{Neu}}^{h}$ and subtracted it from $\mathbf{p}_{h}$, we can write the Galerkin equations as an equivalent problem in

$$
\mathbf{H}_{h, \Sigma}^{\mathrm{O}} \times L_{h}^{\mathrm{O}} \times X_{h} \cong \mathbf{H}_{h} \times L_{h} \times X_{h} \subset \mathbf{H}(\operatorname{div}, \Omega) \times L^{2}(\Omega) \times H_{0}^{1 / 2}(\Gamma) .
$$

The isomorphism is just the extension by zero to the interior of the obstacle $\Omega_{\text {obs }}$; cf. (40) and (41). Proposition 16 can now be used to show stability of the problem with homogeneous boundary conditions and a standard argument for Galerkin methods with nonhomogeneous side conditions proves the estimate (39) from the stability estimate (34).

The Céa estimate (39) can be somewhat simplified by assuming the following hypothesis: there exists a right inverse (a lifting operator) of $\gamma_{\boldsymbol{\nu}}^{\Sigma}: \mathbf{H}_{h}^{\mathrm{O}} \rightarrow \gamma_{\boldsymbol{\nu}}^{\Sigma} \mathbf{H}_{h}^{\mathrm{O}}$, which is bounded as an operator from $H^{-1 / 2}(\Sigma)$ to $\mathbf{H}(\operatorname{div}, \mathrm{O})$, with bound independent of $h$. If this happens, the term related to approximation of $\mathbf{p}$ in (39) can be replaced by

$$
\left\|\beta_{\mathrm{Neu}}-\beta_{\mathrm{Neu}}^{h}\right\|_{-1 / 2, \Sigma}+\inf _{\mathbf{q}_{h} \in \mathbf{H}_{h}^{\mathrm{O}}}\left\|\mathbf{p}-\mathbf{q}_{h}\right\|_{\mathrm{div}, \mathrm{O}}
$$

See [11] for a proof of this result in a very general context. The constant $C$ in this modified version of the Céa estimate then depends on $C_{0}$ and on the bound for the right inverse of the discrete normal trace operator.

A coupling with a direct formulation can be approached in exactly the same terms. There is no compatibility condition for the data of the transmission problem, the solution is defined up to an element of $(\mathbf{0}, 1,1) \in \mathbf{H}(\operatorname{div}, \mathrm{O}) \times L^{2}(\mathrm{O}) \times H^{1 / 2}(\Gamma)$, uniqueness can be enforced by using a restricted formulation (essentially a transpose of the restricted formulation for the indirect coupling) and the correct interior solution is recovered with the same simple postprocessing step of Theorem 4. 


\section{Dirichlet BOUNDARY CONDITIONS}

Let us consider again the geometric setting of Section 5, depicted in Figure 1]. The transmission problem is now

$$
\begin{array}{ll}
\gamma^{\Sigma} u=\beta_{\text {Dir }}, & \\
\mathbf{p}-\nabla u=0 \quad \text { in } \mathrm{O}, & -\operatorname{div} \mathbf{p}=f \quad \text { in } \mathrm{O}, \\
{[\gamma u]=\beta_{0},} & \gamma_{\boldsymbol{\nu}} \mathbf{p}=\partial_{\nu}^{e} u+\beta_{1}, \\
-\Delta u=0 \quad \text { in } \Omega^{e}, & \\
u=\mathcal{O}\left(|\mathbf{x}|^{-1}\right) \text { as }|\mathbf{x}| \rightarrow \infty, &
\end{array}
$$

for a given $\beta_{\text {Dir }} \in H^{1 / 2}(\Sigma)$. Existence and uniqueness of the resulting transmission problem can be deduced using a coupled formulation with the interior problem in primal form and single layer potentials as in [27.

It is relatively simple to check that if we try the analysis of Section 4 , the proofs break down when we arrive at Proposition 15, because when div $\mathbf{p}=0$ in $\mathrm{O}$,

$$
\left\langle\gamma_{\nu} \mathbf{p}, \gamma u^{*}\right\rangle=\left(\mathbf{p}, \nabla u^{*}\right)_{\mathrm{O}}-\left\langle\gamma_{\nu}^{\Sigma} \mathbf{p}, \gamma u^{*}\right\rangle_{\Sigma}
$$

The additional boundary term on $\Sigma$ disappears for Neumann boundary conditions but has to be kept for Dirichlet boundary conditions, and ellipticity of the bilinear form in Proposition 15] is lost. In other words, the simple extension by zero to the interior of the obstacle that worked for Neumann boundary conditions cannot be used here. The result can be recovered by using a different exterior elliptic equation, $-\Delta u+c^{2} u=0$, for some suitable value of $c$. From the point of view of the Laplace equation we will see that the loss is minor: stability of Galerkin approximation will be reached asymptotically, for $h$ small enough, instead of being guaranteed from the beginning.

6.1. Coupling with a direct method: formulation. In the case of problem (42), the data do not give information that allow us to decide whether we can write $u=\mathrm{D} \xi$ in $\Omega^{e}$, and we have to abandon the idea of an indirect formulation. Applying the ideas that lead to (7) (with $\psi=\gamma^{e} u$ ) produces the following system: find $(\mathbf{p}, u, \psi) \in \mathbf{H}(\operatorname{div}, \mathrm{O}) \times L^{2}(\Omega) \times H^{1 / 2}(\Gamma)$ such that

$$
\begin{array}{ll}
(\mathbf{p}, \mathbf{q})_{\mathrm{O}}+(u, \operatorname{div} \mathbf{q})_{\mathrm{O}}-\left\langle\gamma_{\boldsymbol{\nu}} \mathbf{q}, \psi\right\rangle=\ell_{1}(\mathbf{q}), & \forall \mathbf{q} \in \mathbf{H}(\operatorname{div}, \mathrm{O}), \\
(\operatorname{div} \mathbf{p}, v)_{\mathrm{O}}=\ell_{2}(v), & \forall v \in L^{2}(\Omega), \\
\left\langle\left(\frac{1}{2} \mathrm{I}+\mathrm{K}\right)^{t} \gamma_{\nu} \mathbf{p}, \varphi\right\rangle+\langle\mathrm{W} \psi, \varphi\rangle=\ell_{3}(\varphi), & \forall \varphi \in H^{1 / 2}(\Gamma),
\end{array}
$$

where

$\ell_{1}(\mathbf{q}):=\left\langle\gamma_{\boldsymbol{\nu}} \mathbf{q}, \beta_{0}\right\rangle+\left\langle\gamma_{\boldsymbol{\nu}}^{\Sigma} \mathbf{q}, \beta_{\mathrm{Dir}}\right\rangle_{\Sigma}, \quad \ell_{2}(v):=-(f, v)_{\mathrm{O}}, \quad \ell_{3}(\varphi):=\left\langle\left(\frac{1}{2} \mathrm{I}+\mathrm{K}\right)^{t} \beta_{1}, \varphi\right\rangle$.

However, this system is no longer practical because it has introduced an undesirable one-dimensional kernel that is not as simple as the one of the problem set in free space. This kernel is easily described in terms of the function $u_{\Sigma} \in W^{1}\left(\mathbb{R}^{3}\right)$ which satisfies

$$
-\Delta u_{\Sigma}=0 \quad \text { in } \mathbb{R}^{3} \backslash \Sigma, \quad \quad \gamma^{\Sigma} u_{\Sigma}=1 .
$$

Then the function $\left(-\nabla u_{\Sigma}, 1-u_{\Sigma}, 1-\gamma u_{\Sigma}\right)$ can be shown to span the kernel of the operator associated to the equation (43), with a very similar proof to that 
of Proposition [6. This kernel function corresponds to a particular solution of the transmission problem, namely, to the function

$$
u=-\mathrm{D} 1-u_{\Sigma},
$$

which satisfies

$$
\gamma^{\Sigma} u=0, \quad[\gamma u]=1, \quad\left[\partial_{\nu} u\right]=0 .
$$

In order to eliminate this kernel function, we will impose the condition

$$
\langle 1,[\gamma u]\rangle=\left\langle 1, \beta_{0}\right\rangle
$$

as a side condition. Nevertheless, the current formulation does not give access to $\gamma u$ (the mixed formulation takes $u \in L^{2}(\mathrm{O})$, which does not allow us to take its trace on $\Gamma$ ). This imposes the first modification: the boundary unknown will now be

$$
\psi:=\gamma u
$$

In principle, this only changes the right-hand side of (43) as well as the integral representations in the exterior domain, which becomes

$$
u=\mathrm{D}\left(\psi-\beta_{0}\right)-\mathrm{S}\left(\gamma_{\boldsymbol{\nu}} \mathbf{p}-\beta_{1}\right) \quad \text { in } \Omega^{e} .
$$

Condition (44) can now be written in terms of data and unknowns of the system as

$$
\left\langle\gamma_{\nu} \mathbf{p}-\beta_{1}, \phi_{0}\right\rangle+\left\langle\phi_{1}, \psi-\beta_{0}\right\rangle=0
$$

where

$$
\phi_{0}:=\mathrm{V} 1, \quad \phi_{1}:=\left(\frac{1}{2} \mathrm{I}-\mathrm{K}\right)^{t} 1
$$

(recall (14)). This follows from applying the jump relations to the integral representation (45) and plugging the result into (44). This finally leads to the coupled formulation: find $(\mathbf{p}, u, \psi) \in \mathbf{H}(\operatorname{div}, \mathrm{O}) \times L^{2}(\Omega) \times H^{1 / 2}(\Gamma)$ such that

$$
\begin{array}{ll}
(\mathbf{p}, \mathbf{q})_{\mathrm{O}}+(u, \operatorname{div} \mathbf{q})_{\mathrm{O}}-\left\langle\gamma_{\boldsymbol{\nu}} \mathbf{q}, \psi\right\rangle=\ell_{1}(\mathbf{q}), & \forall \mathbf{q} \in \mathbf{H}(\operatorname{div}, \mathrm{O}), \\
(\operatorname{div} \mathbf{p}, v)_{\mathrm{O}}=\ell_{2}(v), & \forall v \in L^{2}(\Omega), \\
\left\langle\left(\frac{1}{2} \mathrm{I}+\mathrm{K}\right)^{t} \gamma_{\nu} \mathbf{p}, \varphi\right\rangle+\langle\mathrm{W} \psi, \varphi\rangle=\ell_{3}(\varphi), & \forall \varphi \in H_{0}^{1 / 2}(\Gamma), \\
\left\langle\gamma_{\boldsymbol{\nu}} \mathbf{p}, \phi_{0}\right\rangle+\left\langle\phi_{1}, \psi\right\rangle=m, &
\end{array}
$$

where

$\ell_{1}(\mathbf{q}):=\left\langle\gamma_{\nu}^{\Sigma} \mathbf{q}, \beta_{\text {Dir }}\right\rangle_{\Sigma}, \quad \ell_{2}(v):=-(f, v)_{\mathrm{O}}, \quad \ell_{3}(\varphi):=\left\langle\left(\frac{1}{2} \mathrm{I}+\mathrm{K}\right)^{t} \beta_{1}, \varphi\right\rangle+\left\langle\mathrm{W} \varphi, \beta_{0}\right\rangle$,

and

$$
m:=\left\langle\beta_{1}, \phi_{0}\right\rangle+\left\langle\phi_{1}, \beta_{0}\right\rangle .
$$

Note the additional asymmetry of (46), stemming from the fact that we test the continuity of flux only in $H_{0}^{1 / 2}(\Gamma)$, whereas we look for $\psi$ in the full $H^{1 / 2}(\Gamma)$. This defect is compensated by the additional scalar condition at the end of (46). 
6.2. Some technical ingredients. We start this section with the technical result on which everything else hinges. Note that the inherent change of operator from the Laplacian to a Yukawa type operator $u \mapsto-\Delta u+c^{2} u$ avoids the use of weights for the Sobolev spaces.

In the following technical lemma, we will use the symbol $\|\cdot\|_{\infty, O}$ to denote the norm of $L^{\infty}(\mathrm{O})$ as well as that of $\left(L^{\infty}(\mathrm{O})\right)^{3}$.

Lemma 19. Let $w \in L^{\infty}(\mathrm{O})$ be such that $\|w\|_{\infty, \mathrm{O}} \leq 1, \nabla w \in\left(L^{\infty}(\mathrm{O})\right)^{3}$ and

$$
\frac{2}{3}\|\nabla w\|_{\infty, \mathrm{O}}<c \text {. }
$$

Then there exists $C=C\left(\|\nabla w\|_{\infty, \mathrm{O}} / c\right)<2$ such that

$$
\left(\|\nabla(w v)\|_{\mathrm{O}}^{2}+c^{2}\|w v\|_{\mathrm{O}}^{2}\right)^{1 / 2} \leq C\left(\|\nabla v\|_{\mathrm{O}}^{2}+c^{2}\|v\|_{\mathrm{O}}^{2}\right)^{1 / 2}, \quad \forall v \in H^{1}(\mathrm{O}) .
$$

Proof. We denote momentarily by $\|\cdot\|_{1, \mathrm{O}, c}$ the weighted $H^{1}(\mathrm{O})$ norm that appears in the statement of the lemma. Note that for any norm associated to an inner product and for all $\eta>0$,

$$
\|a+b\|^{2} \leq(1+\eta)\|a\|^{2}+\left(1+\eta^{-1}\right)\|b\|^{2} .
$$

Therefore,

$$
\begin{aligned}
\|w v\|_{1, \mathrm{O}, c}^{2} & \leq c^{2}\|v\|_{\mathrm{O}}^{2}+\|v \nabla w+w \nabla v\|_{\mathrm{O}}^{2} \\
& \leq c^{2}\|v\|_{\mathrm{O}}^{2}+(1+\eta)\|\nabla w\|_{\infty, \mathrm{O}}^{2}\|v\|_{\mathrm{O}}^{2}+\left(1+\eta^{-1}\right)\|\nabla v\|_{\mathrm{O}} \\
& \leq\left(1+\left(\frac{\|\nabla w\|_{\infty, \mathrm{O}}}{c}\right)^{2}(1+\eta)\right) c^{2}\|v\|_{\mathrm{O}}^{2}+\left(1+\eta^{-1}\right)\|\nabla v\|_{\mathrm{O}}^{2} \\
& \leq C^{2}\|v\|_{1, \mathrm{O}, c}^{2}
\end{aligned}
$$

with

$$
C^{2}:=\max \left\{1+\left(\frac{\|\nabla w\|_{\infty, \mathrm{O}}}{c}\right)^{2}(1+\eta), 1+\eta^{-1}\right\} .
$$

Condition (47) is equivalent to the possibility of finding $\eta$ such that

$$
\frac{1}{3}<\eta<\frac{3 c^{2}}{\|\nabla w\|_{\infty, \mathrm{O}}^{2}}-1
$$

which is equivalent to finding $\eta>0$ such that $C<2$. If we take

$$
\eta:=\frac{1}{2}\left(\frac{3 c^{2}}{\|\nabla w\|_{\infty, \mathrm{O}}^{2}}-\frac{2}{3}\right)
$$

and define $C$ accordingly, we have the result.

Proposition 20. Let $\mathbf{V}:=\{\mathbf{q} \in \mathbf{H}(\operatorname{div}, \mathrm{O}): \operatorname{div} \mathbf{q}=0 \quad$ in $\mathrm{O}\}$. Then, the bilinear form

$$
(\mathbf{p}, \mathbf{q})_{\mathrm{O}}-\left\langle\gamma_{\boldsymbol{\nu}} \mathbf{q}, \gamma^{e} u^{*}\right\rangle-\left\langle\gamma_{\boldsymbol{\nu}} \mathbf{p},\left[\gamma v^{*}\right]\right\rangle+\left(\nabla u^{*}, \nabla v^{*}\right)_{\Omega \cup \Omega^{e}}+c^{2}\left(u^{*}, v^{*}\right)_{\Omega \cup \Omega^{e}}
$$

is elliptic in $\mathbf{V} \times H^{1}\left(\Omega \cup \Omega^{e}\right)$ for c large enough.

Proof. Take $w \in L^{\infty}(\mathrm{O})$ such that $0 \leq w \leq 1, \nabla w \in\left(L^{\infty}(\mathrm{O})\right)^{3}$, and such that $w \equiv 1$ in a neighborhood of $\Gamma$ and $w \equiv 0$ in a neighborhood of $\Sigma$. From Lemma 19 it follows that for $c>0$ large enough, there exists $0<C<2$ such that

$$
\|\nabla(w u)\|_{\mathrm{O}} \leq C\left(\|\nabla u\|_{\mathrm{O}}^{2}+c^{2}\|u\|_{\mathrm{O}}^{2}\right)^{1 / 2}, \quad \forall u \in H^{1}(\mathrm{O}) .
$$


If we now take $\mathbf{q}=\mathbf{p}$ and $v^{*}=u^{*}$ in the bilinear form, we obtain

$$
\begin{aligned}
\|\mathbf{p}\|_{\mathrm{O}}^{2}-\left\langle\gamma_{\nu} \mathbf{p},\right. & \left.\gamma u^{*}\right\rangle+\left\|\nabla u^{*}\right\|_{\Omega \cup \Omega^{e}}^{2}+c^{2}\left\|u^{*}\right\|_{\Omega \cup \Omega^{e}}^{2} \\
& =\|\mathbf{p}\|_{\mathrm{O}}^{2}-\left\langle\gamma_{\nu} \mathbf{p}, \gamma\left(w u^{*}\right)\right\rangle+\left\|\nabla u^{*}\right\|_{\Omega \cup \Omega^{e}}^{2}+c^{2}\left\|u^{*}\right\|_{\Omega \cup \Omega^{e}}^{2} \\
& =\|\mathbf{p}\|_{\mathrm{O}}^{2}-\left(\mathbf{p}, \nabla\left(w u^{*}\right)\right)_{\mathrm{O}}+\left\|\nabla u^{*}\right\|_{\Omega \cup \Omega^{e}}^{2}+c^{2}\left\|u^{*}\right\|_{\Omega \cup \Omega^{e}}^{2} \\
& \geq\left(1-\frac{C}{2}\right)\left(\|\mathbf{p}\|_{\mathrm{O}}^{2}+\left\|\nabla u^{*}\right\|_{\Omega \cup \Omega^{e}}^{2}+c^{2}\left\|u^{*}\right\|_{\Omega \cup \Omega^{e}}^{2}\right)
\end{aligned}
$$

where we have used (48) in the last inequality. Since $(1-C / 2)>0$, we have ellipticity of the bilinear form.

Let $\mathrm{K}_{c}$ and $\mathrm{W}_{c}$ be the boundary integral operators that correspond to taking $\Phi_{c}(r):=\exp (-c r) /(4 \pi r)$ as a fundamental solution. Let

$$
\mathbf{H}_{h} \subset \mathbf{H}(\operatorname{div}, \mathrm{O}), \quad L_{h} \subset L^{2}(\mathrm{O}), \quad X_{h} \subset H^{1 / 2}(\Gamma)
$$

be arbitrary closed subspaces satisfying

$$
\operatorname{div} \mathbf{H}_{h} \subset L_{h}, \quad \sup _{\mathbf{0} \neq \mathbf{q}_{h} \in \mathbf{H}_{h}} \frac{\left(\operatorname{div} \mathbf{q}_{h}, v_{h}\right)_{\mathrm{O}}}{\left\|\mathbf{q}_{h}\right\|_{\operatorname{div}, \mathrm{O}}} \geq C_{0}\left\|v_{h}\right\|_{\mathrm{O}}, \quad \forall v_{h} \in L_{h} .
$$

As a consequence of Proposition 20 we have a very general invertibility result, in parallel to Proposition 17.

Proposition 21. Let $c>0$ be such that Proposition 20 holds. Let $\mathbf{H}_{h}, L_{h}$ and $X_{h}$ be closed subspaces (49) satisfying (50). Then for all $\ell_{1, h} \in \mathbf{H}_{h}^{\prime}, \ell_{2, h} \in X_{h}^{\prime}, \ell_{3, h} \in$ $L_{h}^{\prime}$, the following problem admits a unique solution: find $\left(\mathbf{p}_{h}, \xi_{h}, u_{h}\right) \in \mathbf{H}_{h} \times X_{h} \times L_{h}$ such that

$$
\begin{array}{ll}
\left(\mathbf{p}_{h}, \mathbf{q}_{h}\right)_{\mathrm{O}}-\left\langle\gamma_{\nu} \mathbf{q}_{h},\left(\frac{1}{2} \mathrm{I}+\mathrm{K}_{c}\right) \xi_{h}\right\rangle+\left(u_{h}, \operatorname{div} \mathbf{q}_{h}\right)_{\mathrm{O}}=\ell_{1, h}\left(\mathbf{q}_{h}\right), & \forall \mathbf{q}_{h} \in \mathbf{H}_{h}, \\
\left\langle\gamma_{\boldsymbol{\nu}} \mathbf{p}_{h}, \varphi\right\rangle+\left\langle\mathrm{W}_{c} \xi_{h}, \varphi_{h}\right\rangle=\ell_{2, h}\left(\varphi_{h}\right), & \forall \varphi_{h} \in X_{h}, \\
\left(\operatorname{div} \mathbf{p}_{h}, v_{h}\right)_{\mathrm{O}}=\ell_{3, h}\left(v_{h}\right), & \forall v_{h} \in L_{h},
\end{array}
$$

Moreover, there exists $C>0$ which depends only on $C_{0}$ in (50) (and on c) and is otherwise independent of the choice of the spaces, such that

$$
\left\|\mathbf{p}_{h}\right\|_{\text {div }, \mathrm{O}}+\left\|\xi_{h}\right\|_{1 / 2, \Gamma}+\left\|u_{h}\right\|_{\mathrm{O}} \leq C\left(\left\|\ell_{1, h}\right\|_{\mathbf{H}_{h}^{\prime}}+\left\|\ell_{2, h}\right\|_{X_{h}^{\prime}}+\left\|\ell_{3, h}\right\|_{L_{h}^{\prime}}\right) \text {. }
$$

Proof. Thanks to the conditions of (50) we can limit our study to working on the kernel of the last equation. We define

$$
\mathbf{V}_{h}:=\left\{\mathbf{q}_{h} \in \mathbf{H}_{h}: \operatorname{div} \mathbf{q}_{h}=0 \quad \text { in } \mathrm{O}\right\} \subset \mathbf{V}
$$

(see the definition of $\mathbf{V}$ in the statement of Proposition 20). For any $\jmath_{1, h} \in \mathbf{V}_{h}^{\prime}$ and $\jmath_{2, h} \in X_{h}^{\prime}$, the problem: find $\left(\mathbf{p}_{h}, \xi_{h}\right) \in \mathbf{V}_{h} \times X_{h}$ such that

$$
\begin{array}{ll}
\left(\mathbf{p}_{h}, \mathbf{q}_{h}\right)_{\mathrm{O}}-\left\langle\gamma_{\boldsymbol{\nu}} \mathbf{q}_{h},\left(\frac{1}{2} \mathrm{I}+\mathrm{K}_{c}\right) \xi_{h}\right\rangle=\jmath_{1, h}\left(\mathbf{q}_{h}\right), & \forall \mathbf{q}_{h} \in \mathbf{V}_{h}, \\
\left\langle\gamma_{\nu} \mathbf{p}_{h}, \varphi\right\rangle+\left\langle\mathrm{W}_{c} \xi_{h}, \varphi_{h}\right\rangle=\jmath_{2, h}\left(\varphi_{h}\right), & \forall \varphi_{h} \in X_{h},
\end{array}
$$

is equivalent to: find $\left(\mathbf{p}_{h}, u^{*}\right) \in \mathbf{V}_{h} \times W_{h}$ such that

$$
\begin{array}{ll}
\left(\mathbf{p}_{h}, \mathbf{q}_{h}\right)_{\mathrm{O}}-\left\langle\gamma_{\boldsymbol{\nu}} \mathbf{q}_{h}, \gamma^{e} u^{*}\right\rangle=\jmath_{1, h}\left(\mathbf{q}_{h}\right), & \forall \mathbf{q}_{h} \in \mathbf{V}_{h}, \\
\left\langle\gamma_{\nu} \mathbf{p}_{h},\left[\gamma v^{*}\right]\right\rangle-\left(\nabla u^{*}, \nabla v^{*}\right)_{\Omega \cup \Omega^{e}}-c^{2}\left(u^{*}, v^{*}\right)_{\Omega \cup \Omega^{e}}=\jmath_{2, h}\left(\left[\gamma v^{*}\right]\right), & \forall v^{*} \in W_{h},
\end{array}
$$

where $W_{h}:=\left\{u^{*} \in H^{1}\left(\Omega \cup \Omega^{e}\right):\left[\gamma u^{*}\right] \in X_{h}\right\}$. The equivalence of these two problems is shown by following step by step the proofs of Propositions 13 and 14 . The relation between the unknowns is simple: $\xi_{h}=\left[\gamma u^{*}\right]$ and $u^{*}=\mathrm{D}_{c} \xi_{h}\left(\mathrm{D}_{c}\right.$ is the 
double layer potential associated to the fundamental solution $\Phi_{c}$.) This equivalence holds true for all values of $c>0$. Moreover, problem (54) is elliptic (Proposition 201), which gives uniform invertibility of (53). Finally, (52) follows from this last property and (50), using the standard theory of mixed problems once more.

\subsection{Continuous and discrete analysis.}

Theorem 22. Problem (46) is well posed.

Proof. It is very simple to prove that the well posedness of (46) is equivalent to the well posedness of: find $(\mathbf{p}, u, \psi, n) \in \mathbf{H}(\operatorname{div}, \mathrm{O}) \times L^{2}(\mathrm{O}) \times H^{1 / 2}(\Gamma) \times \mathbb{R}$ such that

$$
\begin{array}{ll}
(\mathbf{p}, \mathbf{q})_{\mathrm{O}}+(u, \operatorname{div} \mathbf{q})_{\mathrm{O}}-\left\langle\gamma_{\boldsymbol{\nu}} \mathbf{q}, \psi\right\rangle=\ell_{1}(\mathbf{q}), & \forall \mathbf{q} \in \mathbf{H}(\operatorname{div}, \mathrm{O}), \\
(\operatorname{div} \mathbf{p}, v)_{\mathrm{O}}=\ell_{2}(v), & \forall v \in L^{2}(\mathrm{O}), \\
\left\langle\left(\frac{1}{2} \mathrm{I}+\mathrm{K}\right)^{t} \gamma_{\nu} \mathbf{p}, \varphi\right\rangle+\langle\mathrm{W} \psi, \varphi\rangle+n \mathrm{~J} \varphi=\ell_{3}(\varphi), & \forall \varphi \in H^{1 / 2}(\Gamma), \\
\left\langle\gamma_{\nu} \mathbf{p}, \phi_{0}\right\rangle+\left\langle\phi_{1}, \psi\right\rangle=m . &
\end{array}
$$

Note that if $\ell_{3}(1)=0$, then the solution of (55) satisfies $n=0$ and solves (46). Now take $c>0$ as in Proposition 20] and consider the integral operators $\mathrm{K}_{c}$ and $\mathrm{W}_{c}$ associated to the fundamental solution $\Phi_{c}$. Then the following problem: find $(\mathbf{p}, u, \psi, n) \in \mathbf{H}(\operatorname{div}, \mathrm{O}) \times L^{2}(\mathrm{O}) \times H^{1 / 2}(\Gamma) \times \mathbb{R}$ such that

$$
\begin{array}{ll}
(\mathbf{p}, \mathbf{q})_{\mathrm{O}}+(u, \operatorname{div} \mathbf{q})_{\mathrm{O}}-\left\langle\gamma_{\boldsymbol{\nu}} \mathbf{q}, \psi\right\rangle=\ell_{1}(\mathbf{q}), & \forall \mathbf{q} \in \mathbf{H}(\operatorname{div}, \mathrm{O}), \\
(\operatorname{div} \mathbf{p}, v)_{\mathrm{O}}=\ell_{2}(v), & \forall v \in L^{2}(\mathrm{O}), \\
\left\langle\left(\frac{1}{2} \mathrm{I}+\mathrm{K}_{c}\right)^{t} \gamma_{\nu} \mathbf{p}, \varphi\right\rangle+\left\langle\mathrm{W}_{c} \psi, \varphi\right\rangle=\ell_{3}(\varphi), & \forall \varphi \in H^{1 / 2}(\Gamma), \\
n=m, &
\end{array}
$$

is a compact perturbation of (55): the differences $\mathrm{K}-\mathrm{K}_{c}$ and $\mathrm{W}-\mathrm{W}_{c}$ are compact and all other operators we have changed are of finite rank.

We now take $\mathbf{H}_{h}=\mathbf{H}(\operatorname{div}, \mathrm{O}), L_{h}=L^{2}(\mathrm{O})$ and $X_{h}=H^{1 / 2}(\Gamma)$ in Proposition 21 and note that conditions (501) are satisfied by these spaces. Transposing and changing signs of the third row and columns, we obtain well posedness of the set of the first three equations of (56). Therefore, (56) is well posed and the operator associated to (55) is Fredholm of index zero.

We finally want to prove that the kernel of the operator associated to equations (55) is trivial. If $\left(\mathbf{p}_{0}, u_{0}, \psi_{0}, n_{0}\right)$ satisfies these equations with zero right-hand side, it follows readily that $n_{0}=0$ (test with $\varphi=1$ ). Taking $u_{0}:=\mathrm{D} \psi_{0}-\mathrm{S} \gamma_{\nu} \mathbf{p}_{0}$ in $\Omega^{e}$ we easily prove that

$$
\nabla u_{0}=\mathbf{p}_{0} \quad \text { in } \mathrm{O}, \quad-\Delta u_{0}=0 \quad \text { in } \mathrm{O} \cup \Omega^{e},
$$

as well as

$$
\gamma^{\Sigma} u_{0}=0, \quad \gamma u_{0}=\psi_{0}, \quad \gamma_{\nu} \mathbf{p}_{0}=\partial_{\nu}^{e} u_{0}
$$

Using Green's Third Identity we can identify $\mathrm{D} \psi_{0}-\mathrm{S} \partial_{\nu}^{e} u_{0}=u_{0}=\mathrm{D} \gamma^{e} u_{0}-\mathrm{S} \partial_{\nu}^{e} u_{0}$ and by (19), there exists $c \in \mathbb{R}$ such that $\psi_{0}=\gamma^{e} u_{0}+c$. However, the last equation of the set (46)) (with $m=0$ ) is

$$
0=\left\langle 1,\left(\frac{1}{2} \mathrm{I}-\mathrm{K}\right) \psi_{0}+\mathrm{V} \gamma_{\nu} \mathbf{p}_{0}\right\rangle=\left\langle 1, \psi_{0}-\gamma^{e} u_{0}\right\rangle,
$$

from where $\left[\gamma u_{0}\right]=0$. The pair $\left(u_{0}, \mathbf{p}_{0}\right)$ then solves the homogeneous transmission problem and therefore $u_{0}=0, \mathbf{p}_{0}=0$ and $\psi_{0}=0$. 
Discretization of equations (46) is achieved with the three sequences of finite dimensional subspaces

$$
\mathbf{H}_{h} \subset \mathbf{H}(\operatorname{div}, \mathrm{O}), \quad L_{h} \subset L^{2}(\mathrm{O}), \quad \mathbb{P}_{0} \subset X_{h} \subset H^{1 / 2}(\Gamma) .
$$

We assume conditions (50) for $\mathbf{H}_{h}$ and $L_{h}$, with $C_{0}$ independent of $h$. The assumption $\mathbb{P}_{0} \subset X_{h}$, allows us to separate

$$
X_{h}=\mathbb{P}_{0} \oplus X_{h}^{0}, \quad X_{h}^{0} \subset H_{0}^{1 / 2}(\Gamma) .
$$

The proposed discretization of (46) is: find $\left(\mathbf{p}_{h}, u_{h}, \psi_{h}\right) \in \mathbf{H}_{h} \times L_{h} \times X_{h}$ such that

$$
\begin{array}{ll}
\left(\mathbf{p}_{h}, \mathbf{q}_{h}\right)_{\mathrm{O}}+\left(u_{h}, \operatorname{div} \mathbf{q}_{h}\right)_{\mathrm{O}}-\left\langle\gamma_{\boldsymbol{\nu}} \mathbf{q}_{h}, \psi_{h}\right\rangle=\ell_{1}\left(\mathbf{q}_{h}\right), & \forall \mathbf{q}_{h} \in \mathbf{H}_{h}, \\
\left(\operatorname{div} \mathbf{p}_{h}, v_{h}\right)_{\mathrm{O}}=\ell_{2}\left(v_{h}\right), & \forall v_{h} \in L_{h}^{2}, \\
\left\langle\left(\frac{1}{2} \mathrm{I}+\mathrm{K}\right)^{t} \gamma_{\nu} \mathbf{p}_{h}, \varphi_{h}\right\rangle+\left\langle\mathrm{W} \psi_{h}, \varphi_{h}\right\rangle=\ell_{3}\left(\varphi_{h}\right), & \forall \varphi_{h} \in X_{h}^{0}, \\
\left\langle\gamma_{\nu} \mathbf{p}_{h}, \phi_{0}\right\rangle+\left\langle\phi_{1}, \psi_{h}\right\rangle=m . &
\end{array}
$$

Let

$$
\varepsilon_{h}(\mathbf{p}, u, \psi):=\inf _{\mathbf{q}_{h} \in \mathbf{H}_{h}}\left\|\mathbf{p}-\mathbf{q}_{h}\right\|_{\text {div }, \mathrm{O}}+\inf _{v_{h} \in L_{h}}\left\|u-v_{h}\right\|_{\mathrm{O}}+\inf _{\varphi_{h} \in X_{h}}\left\|\psi-\varphi_{h}\right\|_{1 / 2, \Gamma} .
$$

We also assume that

$$
\lim _{h \rightarrow 0} \varepsilon_{h}(\mathbf{p}, u, \psi)=0, \quad \forall(\mathbf{p}, u, \psi) \in \mathbf{H}(\operatorname{div}, \mathrm{O}) \times L^{2}(\mathrm{O}) \times H^{1 / 2}(\Gamma) .
$$

Theorem 23. If the discrete spaces (57) satisfy (50) and the approximation property (59), then problem (58) has a unique solution for $h$ small enough. Moreover, there exist $h_{0}$ and $C>0$ such that for $h \leq h_{0}$,

$$
\left\|\mathbf{p}-\mathbf{p}_{h}\right\|_{\text {div }, \mathrm{O}}+\left\|u-u_{h}\right\|_{\mathrm{O}}+\left\|\psi-\psi_{h}\right\|_{1 / 2, \Gamma} \leq C \varepsilon_{h}(\mathbf{p}, u, \psi) .
$$

The constant $C$ depends only on $C_{0}>0$ (and on the continuous problem) but $h_{0}$ depends on the sequence of discrete spaces.

Proof. Unique solvability and stability of the discrete operator associated to the equations (58) is equivalent to the same properties for a Galerkin discretization of (55) with $\mathbf{H}_{h} \times L_{h} \times X_{h} \times \mathbb{R}$ as a discrete space.

Proposition 21 proves that any Galerkin discretization of (56) is stable as long as the discrete spaces satisfy (50) and that the only dependence of the stability on the discrete spaces is through $C_{0}$ on (50). Galerkin stability and the approximation property (59) imply convergence of the method, a property that is transferred to invertible compact perturbations of the operator equations (see for instance [21. Chapter 13]). The stability estimate for the compact perturbation (which is equivalent to the Céa estimate (60) ) uses a constant that is proportional to that of the unperturbed discrete problem: the proportionality factor depends only on the operator and not on the discrete spaces.

\section{NUMERICAL EXPERIMENTS}

In all of our examples we will be using tetrahedral meshes for the interior domain and the inherited triangular meshes for the boundary element discretization. The discrete spaces will be the first order Raviart-Thomas finite element (see [3]) to approximate the flux variable $\mathbf{p}$, piecewise constant functions for the discrete version of $u$ and the standard first order Lagrange finite element method for the boundary unknown $\psi$. It is well known that conditions (17) and (18) are satisfied 
for these choices of $\mathbf{H}_{h}$ and $L_{h}$. The boundary integrals are approximated using the techniques of $[12$.

In the sequel, $N$ stands for the number of degrees of freedom defining the finite element spaces $\mathbf{H}_{h}, X_{h}$ and $M_{h}$ and the individual relative errors are given by

$$
e(\mathbf{p}):=\frac{\left\|\mathbf{p}-\mathbf{p}_{h}\right\|_{\operatorname{div}, \Omega}}{\|\mathbf{p}\|_{\operatorname{div}, \Omega}}, \quad e(u):=\frac{\left\|u-u_{h}\right\|_{0, \Omega}}{\|u\|_{0, \Omega}}
$$

and

$$
e(\psi):=\frac{\left\|\psi-\psi_{h}\right\|_{0, \Gamma}^{1 / 2}\left\|\psi-\psi_{h}\right\|_{1, \Gamma}^{1 / 2}}{\|\psi\|_{0, \Gamma}^{1 / 2}\|\psi\|_{1, \Gamma}^{1 / 2}} .
$$

Also, we let $r(\mathbf{p}), r(u)$ and $r(\psi)$ be the experimental rates of convergence given by

$$
r(\mathbf{p}):=\frac{\log \left(e(\mathbf{p}) / e^{\prime}(\mathbf{p})\right)}{\log \left(h / h^{\prime}\right)}, \quad r(u):=\frac{\log \left(e(u) / e^{\prime}(u)\right)}{\log \left(h / h^{\prime}\right)} \quad r(\psi):=\frac{\log \left(e(\psi) / e^{\prime}(\psi)\right)}{\log \left(h / h^{\prime}\right)},
$$

where $h$ and $h^{\prime}$ denote two consecutive meshsizes with corresponding errors $e$ and $e^{\prime}$.

We first present an example illustrating the performance of the nonsymmetric mixed FEM and BEM method applied to the direct formulation (12) of problem (11) on a set of irregular shape-uniform tetrahedral meshes of the cube $\Omega:=(-1,1)^{3}$. The function $u: \Omega \cup \Omega^{e} \rightarrow \mathbb{R}$ given by

$$
u(\mathbf{x}):=\left\{\begin{array}{l}
\cos \left(\pi x_{1}\right) \cos \left(\pi x_{2}\right) \cos \left(\pi x_{3}\right) \text { in } \Omega \\
1 /(4|\mathbf{x}|) \quad \text { in } \Omega^{e}
\end{array}\right.
$$

is a solution of our model problem if suitable nonhomogeneous transmission conditions are considered on $\Gamma$.

TABle 2. Degrees of freedom, relative errors and rates of convergence (EXAMPLE 1)

\begin{tabular}{c||c|c||c|c||c|c}
$N$ & $e(\mathbf{p})$ & $r(\mathbf{p})$ & $e(u)$ & $r(u)$ & $e(\psi)$ & $r(\psi)$ \\
\hline \hline 8593 & 0.278273 & - & 0.279434 & - & 0.066873 & - \\
11578 & 0.250620 & 1.0531 & 0.251197 & 1.0719 & 0.058907 & 1.2762 \\
18838 & 0.210811 & 1.0661 & 0.210816 & 1.0801 & 0.046163 & 1.5024 \\
30698 & 0.179779 & 0.9783 & 0.179627 & 0.9836 & 0.039395 & 0.9739 \\
40339 & 0.164711 & 0.9615 & 0.164499 & 0.9664 & 0.035875 & 1.0283 \\
81854 & 0.127082 & 1.0996 & 0.126785 & 1.1040 & 0.029287 & 0.8602
\end{tabular}

In Table 2 we summarize the convergence history for a sequence of uniform meshes of the computational domain $\bar{\Omega}$. The results show clearly that the linear convergence provided by the Céa estimate in Theorem 5 (which is also valid for the direct scheme) and standard interpolation error estimates (see [3]) is attained for all unknowns.

In the second example we need to test the performance of scheme (38). The computational domain is now $\Omega:=(-1,1)^{3} \backslash\left[-\frac{1}{2}, \frac{1}{2}\right]^{3}$ and Neumann boundary conditions are imposed on the internal boundary $\Sigma$. We still consider the same exact solution (61) and the same discretisation spaces (after incorporating the new boundary condition on $\Sigma$ to the first order Raviart-Thomas space). We recall that 
we are using here an indirect formulation, consequently, in Table 3 we do not present numerical results for the boundary unknown since the corresponding exact solution is not known. The rate of convergence in the flux variable $\mathbf{p}$ and the Lagrange multiplier $u$ behave as predicted by Theorem 18 and well-known interpolation error estimates.

TABlE 3. Degrees of freedom, relative errors and rates of convergence (EXAMPLE 2)

\begin{tabular}{c||c|c||c|c}
$N$ & $e(\mathbf{p})$ & $r(\mathbf{p})$ & $e(u)$ & $r(u)$ \\
\hline \hline 3004 & 0.407653 & - & 0.483004 & - \\
8554 & 0.280336 & 1.0734 & 0.303482 & 1.3322 \\
15042 & 0.227212 & 1.1167 & 0.258337 & 0.8560 \\
25911 & 0.195052 & 0.8419 & 0.230108 & 0.6384 \\
42297 & 0.167489 & 0.9327 & 0.203752 & 0.7447 \\
52944 & 0.152555 & 1.2279 & 0.182612 & 0.9742
\end{tabular}

In the third and last example we study the performance of the numerical scheme (58) corresponding to a direct formulation of an exterior Dirichlet problem. Here again we consider the same exact solution (61) on $\Omega:=(-1,1)^{3} \backslash\left[-\frac{1}{2}, \frac{1}{2}\right]^{3}$. It is clear from Table 4 that, as expected, we have a linear asymptotic convergence for all variables.

TABLE 4. Degrees of freedom, relative errors and rates of convergence (EXAMPLE 3)

\begin{tabular}{c||c|c||c|c||c|c}
$N$ & $e(\mathbf{p})$ & $r(\mathbf{p})$ & $e(u)$ & $r(u)$ & $e(\psi)$ & $r(\psi)$ \\
\hline \hline 3094 & 0.407993 & - & 0.417140 & - & 0.326680 & - \\
8750 & 0.280279 & 1.0835 & 0.282473 & 1.1250 & 0.204786 & 1.3467 \\
15392 & 0.227090 & 1.1178 & 0.227860 & 1.1412 & 0.161304 & 1.2678 \\
26523 & 0.194875 & 0.8434 & 0.194781 & 0.8648 & 0.116847 & 1.7775 \\
43185 & 0.167274 & 0.9399 & 0.167093 & 0.9436 & 0.098330 & 1.0618 \\
60434 & 0.145909 & 1.2199 & 0.145527 & 1.2336 & 0.088739 & 0.9162
\end{tabular}

\section{REFERENCES}

[1] R.A. Adams. Sobolev spaces. Pure and Applied Mathematics, Vol. 65. Academic Press, New York, London, 1975. MR0450957 (56:9247)

[2] J. Bielak, R.C. MacCamy, Symmetric finite element and boundary integral coupling methods for fluid-solid interaction. Quart. Appl. Math. 49 (1991), no. 1, 107-119. MR.1096235 (91m:65270)

[3] F. Brezzi, M. Fortin. Mixed and hybrid finite element methods. Springer Series in Computational Mathematics, 15. Springer-Verlag, New York, 1991. MR.1115205 (92d:65187)

[4] F. Brezzi, C. Johnson. On the coupling of boundary integral and finite element methods. Calcolo 16 (1979), 189-201. MR.569615 (81e:65055b)

[5] F. Brezzi, C. Johnson, J.C. Nédélec. On the coupling of boundary integral and finite element methods. Proceedings of the Fourth Symposium on Basic Problems of Numerical Mathematics (Plezň, 1978), 103-114. Charles Univ. Prague, 1978. MR566158(81e:65055a) 
[6] C. Carstensen, S. Funken. Coupling of mixed finite elements and boundary elements. IMA J. Numer. Anal. 20 (2000), 461-480. MR.1773269 (2002e:65171)

[7] C. Carstensen, S. Funken. Coupling of nonconforming finite elements and boundary elements. I. A priori estimates. Computing 62 (1999), 229-241. MR.1697839 (2000h:65168)

[8] B. Cockburn, F.-J. Sayas. Symmetric coupling of boundary element and discontinuous Galerkin methods: algorithms and examples. Submitted.

[9] M. Costabel. Symmetric methods for the coupling of finite elements boundary elements, Boundary elements IX, Vol. 1 (Stuttgart, 1987), 411-420, Comput. Mech., Southampton, 1987. MR965328 (89j:65068)

[10] M. Costabel. Boundary integral operators on Lipschitz domains: elementary results. SIAM J. Math. Anal. 19 (1988), 613-626. MR937473 (89h:35090)

[11] V. Domínguez, F.-J. Sayas. Stability of discrete liftings. C. R. Math. Acad. Sci. Paris 337 (2003) 805-808. MR2033124

[12] S. Erichsen, S.A. Sauter. Efficient automatic quadrature in 3-d Galerkin BEM. Comput. Methods Appl. Mech. Eng. 157 (1998), 215-224. MR.1634288 (99e:65163)

[13] G.N. Gatica, G.C. Hsiao. Boundary-field equation methods for a class of nonlinear problems. Pitman Research Notes in Mathematics Series, 331. Longman, Harlow, 1995. MR1379331 (97k:65269)

[14] G.N. Gatica, G.C. Hsiao, F.-J. Sayas. Relaxing the hypotheses on Bielak-MacCamy's BEMFEM coupling. Submitted.

[15] G.N. Gatica, N. Heuer, F.-J. Sayas. A direct coupling of local discontinuous Galerkin and boundary element methods. Math. Comput. 79 (2010), 1369-1394.

[16] G.N. Gatica, F.-J. Sayas. An a priori error analysis for the coupling of local discontinuous Galerkin and boundary element methods. Math. Comp. 75 (2006), 1675-1696. MR 2240630 (2007e:65119)

[17] V. Girault, P.A. Raviart. Finite element methods for Navier-Stokes equations. Theory and algorithms. Springer Series in Computational Mathematics, 5. Springer-Verlag, Berlin, 1986. MR851383 (88b:65129)

[18] C. Johnson, J.C. Nédélec. On the coupling of boundary integral and finite element methods. Math. Comp. 35 (1980), 1063-1079. MR583487(82c:65072)

[19] H. Han. A New class of variational formulations for the coupling finite and boundary element methods, J. Comp. Math. 8 (1990) 223-232. MR1299224

[20] S. Hildebrandt, E. Wienholtz. Constructive proofs of representation theorems in separable Hilbert space. Comm. Pure Appl. Math. 17 (1964), 369-373. MR0166608 (29:3881)

[21] R. Kress. Linear integral equations. Second edition. Applied Mathematical Sciences, 82. Springer-Verlag, New York, 1999. MR 1723850 (2000h:45001)

[22] A. Laliena, F.-J. Sayas. Theoretical aspects of the application of convolution quadrature to scattering of acoustic waves, Numer. Math. 112 (2009), 637-678. MR2507621

[23] W. McLean. Strongly elliptic systems and boundary integral equations. Cambridge University Press, Cambridge, 2000. MR.1742312 (2001a:35051)

[24] S. Meddahi. An optimal iterative process for the Johnson-Nédélec method of coupling boundary and finite elements. SIAM J. Numer. Anal. 35 (1998), 1393-1415. MR.1620160 (99f:65173)

[25] S. Meddahi, J. Valdés, O. Menéndez, P. Pérez. On the coupling of boundary integral and mixed finite element methods. J. Comput. Appl. Math. 69 (1996), no. 1, 113-124. MR1391614 (97e:65137)

[26] J.C. Nédélec. Acoustic and electromagnetic equations. Integral representations for harmonic problems. Applied Mathematical Sciences, 144. Springer-Verlag, New York, 2001. MR:1822275 (2002c:35003)

[27] F.-J. Sayas. The validity of Johnson-Nédélec's BEM-FEM coupling of polygonal interfaces. SIAM J. Numer. Anal. 47 (2009), 3451-3463. MR2551202

[28] A. Sequeira. The coupling of boundary integral and finite element methods for the bidimensional exterior steady Stokes problem. Math. Methods Appl. Sci. 5 (1983), 356-375. MR7716661 (85g:65121)

[29] O.C. Zienkiewicz, D.W. Kelly, P. Bettess. Marriage à la mode - the best of both worlds (finite elements and boundary integrals). Energy methods in finite element analysis, pp. 81-107, Wiley, Chichester, 1979. MR.537001 (80e:65107) 
Departamento de Matemáticas, Universidad de Oviedo, Calvo Sotelo s/n, 33007 OVIEDO, Spain

E-mail address: salim@uniovi.es

Departamento de Matemática Aplicada, CPS, Universidad de Zaragoza, 50018 Zaragoza, Spain and School of Mathematics, University of Minnesota, Minneapolis, Minnesota 55455

E-mail address: sayas002@umn.edu

Departamento de Matemáticas, Universidad de A Coruña, Facultad de Informática, Campus de Elviña S/n, 15071 A Coruña, Spain

E-mail address: vselgas@udc.es 